\title{
Quantitative Analysis of Doping-Induced Polarons and Charge- Transfer Complexes of Poly(3-hexylthiophene) in Solution
}

\author{
Malavika Arvind, ${ }^{\S}$ Claudia E. Tait, ${ }^{\S}$ Michele Guerrini, Jannis Krumland, Ana M. Valencia, \\ Caterina Cocchi, Ahmed E. Mansour, Norbert Koch, Stephen Barlow, Seth R. Marder, Jan Behrends, \\ and Dieter Neher*
}

Cite This: https://dx.doi.org/10.1021/acs.jpcb.0c03517

Read Online

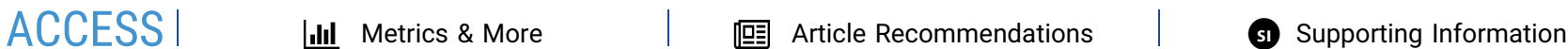

ABSTRACT: The mechanism and the nature of the species formed by molecular doping of the model polymer poly(3hexylthiophene) (P3HT) in its regioregular (rre-) and regiorandom (rra-) forms in solution are investigated for three different dopants: the prototypical $\pi$-electron acceptor 2,3,5,6-tetrafluoro7,7,8,8-tetracyanoquinodimethane ( $\left.\mathrm{F}_{4} \mathrm{TCNQ}\right)$, the strong Lewis acid tris(pentafluorophenyl)borane (BCF), and the strongly oxidizing complex molybdenum tris[1-(methoxycarbonyl)-2(trifluoromethyl)ethane-1,2-dithiolene $]\left(\mathrm{Mo}\left(\mathrm{tfd}-\mathrm{CO}_{2} \mathrm{Me}\right)_{3}\right)$. In a combined optical and electron paramagnetic resonance study, we show that the doping of rreP3HT in solution occurs by integer charge transfer, resulting in formation of $\mathrm{P} 3 \mathrm{HT}$ radical cations

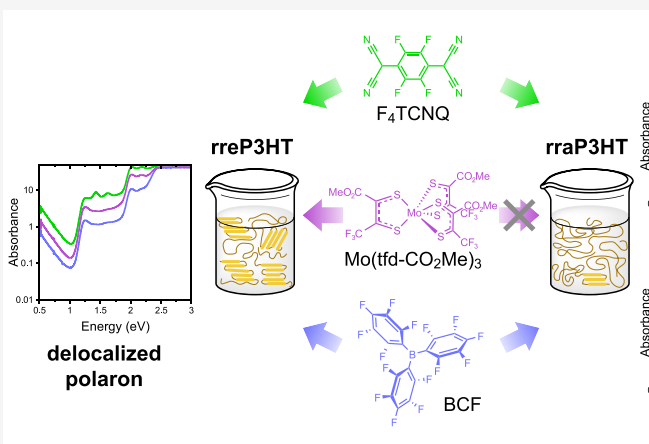
charge-transfer complex (polarons) for all of the dopants considered here. Remarkably, despite the different chemical nature of the dopants and dopant-polymer interaction, the formed polarons exhibit essentially identical optical absorption spectra. The situation is very different for the doping of rraP3HT, where we observe formation of a charge-transfer complex with $\mathrm{F}_{4}$ TCNQ and of a "localized" P3HT polaron on nonaggregated chains upon doping with BCF, while there is no indication of dopant-induced species in the case of $\mathrm{Mo}\left(\mathrm{tfd}-\mathrm{CO}_{2} \mathrm{Me}\right)_{3}$. We estimate the ionization efficiency of the respective dopants for the two polymers in solution and report the molar extinction coefficient spectra of the three different species. Finally, we observe increased spin delocalization in regioregular compared to regiorandom P3HT by electron nuclear double resonance, suggesting that the ability of the charge to delocalize on aggregates of planarized polymer backbones plays a significant role in determining the doping mechanism.

\section{INTRODUCTION}

Organic semiconductors (OSCs) have advantages over inorganic semiconductors in terms of ease of processing, material flexibility, and cost, ${ }^{1}$ but they typically suffer from poor conductivity. Doping offers a solution to this problem, as the charge-carrier density, and thereby the conductivity, can be improved by controlled addition of $\mathrm{p}$ - or $\mathrm{n}$-dopants. ${ }^{2}$ However, stable and reliable doping of OSCs is difficult to achieve, and the mechanism of molecular doping is currently not fully understood for some of the dopants that have been successfully used. Ideally, a conjugated molecule or polymer and a simple molecular one-electron oxidant or reductant (p- or n-dopant respectively) undergo integer charge transfer (ICT), i.e., complete transfer of an electron from the host to the dopant or vice versa. Experimental and theoretical studies during the past years support this scenario in some cases but indicate that in others the interaction of host and dopant can result in formation of a charge-transfer complex (CTC), in which the frontier orbitals of the host and the dopant interact, resulting in a partial charge transfer. ${ }^{3-6}$ With ICT, ionic species are formed directly and, upon their dissociation, the conductivity of the material increases. CTCs, on the other hand, are still electrically neutral, and additional thermal or optical excitation is required to create mobile charge carriers.

One of the most extensively investigated host:dopant systems is regioregular poly(3-hexylthiophene) (rreP3HT) doped with the electron acceptor 2,3,5,6-tetrafluoro-7,7,8,8tetracyanoquinodimethane $\left(\mathrm{F}_{4} \mathrm{TCNQ}\right)$. This system is special in several respects. First, $\mathrm{F}_{4} \mathrm{TCNQ}$ is a planar molecule, allowing for $\pi-\pi$ stacking with the conjugated backbone of the organic semiconductor upon doping. ${ }^{7,8}$ In fact, early X-ray diffraction studies suggested that $\mathrm{F}_{4}$ TCNQ forms a cocrystal with $\mathrm{rreP} 3 \mathrm{HT},{ }^{9,10}$ though more recent studies have revised this

Received: April 20, 2020

Revised: June 22, 2020

Published: June 23, 2020 
picture, suggesting that the $\mathrm{F}_{4} \mathrm{TCNQ}$ anions reside in the aliphatic side-chain regions of the solid doped layer. ${ }^{11,12}$ rreP3HT, on the other hand, stands out because of its large conformational freedom, owing to the smooth variation of the potential energy as a function of the dihedral angle between subsequent thiophene units. ${ }^{13}$ Therefore, if rreP3HT is dissolved in common organic solvents such as chlorobenzene or chloroform, it adopts the twisted conformation of a wormlike chain. A characteristic feature of this state is the broad (Gaussian-like) and featureless UV-vis absorption spectrum, assigned to a distribution of conjugation lengths. ${ }^{14}$ The situation changes drastically when a poor solvent is added, upon which planarization of the polymer backbone accompanied by chain aggregation leads to a red-shifted and wellstructured absorption spectrum, with the $0-0$ transition at around $2 \mathrm{eV}$ and a pronounced vibronic progression. ${ }^{15,16}$ Various groups reported a similar spectral change of the polymer absorption properties upon molecular doping of rreP3HT in solution, ${ }^{8,9}$ potentially driven by the lower solubility of the doped (charged) backbone with its more planar quinoid form in the original solvent. It was proposed that the conformational transition to a more planar backbone and a larger conjugation length are required for efficient ICT, ${ }^{17}$ highlighting the importance of charge delocalization for the mechanism and efficiency of charge transfer. This might also explain why doping of short oligothiophenes with $\mathrm{F}_{4}$ TCNQ results exclusively in CTCs. ${ }^{10}$ In fact, when the ability of the polythiophene polymer backbone to planarize or aggregate is inhibited through steric hindrance, e.g., in regiorandom P3HT (rraP3HT), ICT by $\mathrm{F}_{4}$ TCNQ is strongly suppressed and CTCs are the primary products of doping.

Similar to the optical absorption of neutral P3HT, the spectral shape and position of the doping-induced NIR absorption features depend on the backbone conformation and intermolecular coupling. p-doped conjugated polymers generally exhibit two polaron absorption features, $\mathrm{P}_{2}$ and $\mathrm{P}_{1}$. Recent theoretical studies assigned the high-energy feature $\left(\mathrm{P}_{2}\right)$ to an electronic transition from the highest occupied molecular orbital (HOMO) of an adjacent neutral polymer segment (on the same chain) to the lowest unoccupied molecular orbital (LUMO) of the charged segment, while the low-energy peak $\left(\mathrm{P}_{1}\right)$ was attributed to an electronic transition from the same HOMO to an unoccupied gap state of the cationic unit. ${ }^{18}$ For $\mathrm{F}_{4}$ TCNQ-doped rreP3HT (in aggregated solution and in the solid state), this $\mathrm{P}_{2}$ feature consists of two peaks, with absorption maxima at 1.3 and $1.7 \mathrm{eV}$, and $\mathrm{P}_{1}$ appears as a broad absorption peak at ca. $0.35 \mathrm{eV}$. Davydov splitting has been suggested as the reason for the presence of two closely spaced peaks in the $\mathrm{P}_{2}$ absorption feature. ${ }^{19}$ The exact position and spectral weight of these absorption features depend, however, on the quality of polymer packing and intermolecular interaction, which are in turn determined by the degree of delocalization of the polaron in two dimensions competing with polaron localization due to the presence of the dopant anion. ${ }^{20-23}$ In doped rraP3HT films on the other hand, the absorption of doping-induced polarons is blue-shifted, displaying broad absorptions at $1.65 \mathrm{eV}$ (unsplit $\mathrm{P}_{2}$ band) and at $0.65 \mathrm{eV}\left(\mathrm{P}_{1}\right){ }^{24}$ This suggests that a more localized polaron is formed on individual disordered chains. ${ }^{25}$ The assignment of spectral features to the specific nature of the doping-induced species is, however, challenging in doped P3HT layers. This is due to the multicrystalline morphology of solid rreP3HT layers, where crystalline and amorphous regions coexist and the result of doping also depends on the processing conditions. For example, while most studies in the past reported ICT to prevail in $\mathrm{F}_{4}$ TCNQ-doped rreP3HT films, ${ }^{3,10,26}$ a recent report by Jacobs et al. showed that $\mathrm{F}_{4}$ TCNQ-doped rreP3HT films are dominated by CTCs when processed from higher temperature solutions. ${ }^{27}$ A subsequent study by Neelamraju et al. suggested the coexistence of ICT and CTC states in $\mathrm{F}_{4}$ TCNQ-doped rreP3HT films, with ICT dominating in the crystalline phase and CTC being prevalent in the amorphous regions. ${ }^{28}$ It has also been shown that the doping mechanism in polythiophene films can be varied between ICT or CTC by controlling the degree of interaction between the dopant and the polymer backbone via side-chain functionalization. ${ }^{29}$ Consequently, there is still an ongoing discussion regarding the exact nature of the species observed in doped samples via optical methods. ${ }^{3,26}$

In this work, we combine high-resolution UV-vis-NIR, electron paramagnetic resonance (EPR), and electron nuclear double resonance (ENDOR) spectroscopies to study the nature of the species resulting from p-doping of the model polymer $\mathrm{P} 3 \mathrm{HT}$ in its regioregular (rreP3HT) and regiorandom (rraP3HT) forms in solution. Ab initio calculations based on time-dependent density functional theory (TDDFT) complement our analysis. The two forms of P3HT differ mainly in their ability to aggregate and form ordered structures in solutions and films, thereby providing the opportunity to investigate the effect of intra- and intermolecular order on the prevalent doping mechanism and the absorption properties of the resulting species. By studying the process in solution, we avoid the complexity of the multiphase morphology of solid P3HT films. Because rraP3HT chains always adopt a coiled conformation, doping-induced species in rraP3HT may serve as models for precursor states to polarons on doped rreP3HT, which additionally undergo chain planarization/aggregation upon doping. We consider three types of molecular dopants: the well-known $\mathrm{F}_{4} \mathrm{TCNQ}$ the more three-dimensional molybdenum tris[1-(methoxycarbonyl)-2-(trifluoromethyl)ethane-1,2-dithiolene] $\left(\mathrm{Mo}\left(\mathrm{tfd}-\mathrm{CO}_{2} \mathrm{Me}\right)_{3}\right)$, and the Lewis acid dopant tris(pentafluorophenyl)borane (BCF). ${ }^{30-34}$ The chemical structures and energy levels of the dopants and polymers are shown in Figure $1 \mathrm{a}$ and $1 \mathrm{~b}$, respectively. Compared to the planar $\mathrm{F}_{4} \mathrm{TCNQ}, \mathrm{Mo}\left(\mathrm{tfd}-\mathrm{CO}_{2} \mathrm{Me}\right)_{3}$ exhibits a bulkier structure and a lower electron affinity (EA) $(5.0 \mathrm{eV}$ versus $5.2 \mathrm{eV}) .^{33,35} \mathrm{BCF}$, on the other hand, is not expected to dope P3HT because of its moderate EA (i.e., high LUMO) (ca. $3 \mathrm{eV}) .{ }^{36}$ Despite this, results from UV-vis-NIR and EPR studies on BCF-doped rreP3HT solutions and films suggested ICT with a fairly high ionization efficiency. ${ }^{37}$ Very recently, Yurash et al. reported that doping of the conjugated polymer poly $\left[2,6-\left(4,4-b i s(2-h e x a d e c y l)-4 H\right.\right.$-cyclopenta[2,1-b;3,4- $\left.b^{\prime}\right]$ dithiophene)-alt-4,7(2,1,3-benzothiadiazole)] (PCPDTBT) with $\mathrm{BCF}$ occurs in a multistep process. ${ }^{38}$ Initially, traces of water cause formation of $\mathrm{BCF} \cdot \mathrm{H}_{2} \mathrm{O}$, which has long been known to be a strong Brønsted acid and capable of oxidizing molecules such as ferrocene (the oxidation potential of which is similar to that of reP3HT), presumably with the generation of $\mathrm{H}_{2}{ }^{39}$ In the case of PCPDTBT oxidation, it was suggested that there is an endothermic protonation step which results in a positively charged protonated polymer and a $\mathrm{BCF}-\mathrm{OH}^{-}$ anion, followed by an exothermic electron-transfer step in which a nearby neutral polymer chain (or segment) and the protonated polymer react to form a radical cation (polaron) and a neutral protonated radical species. ${ }^{38}$ As protonation was 

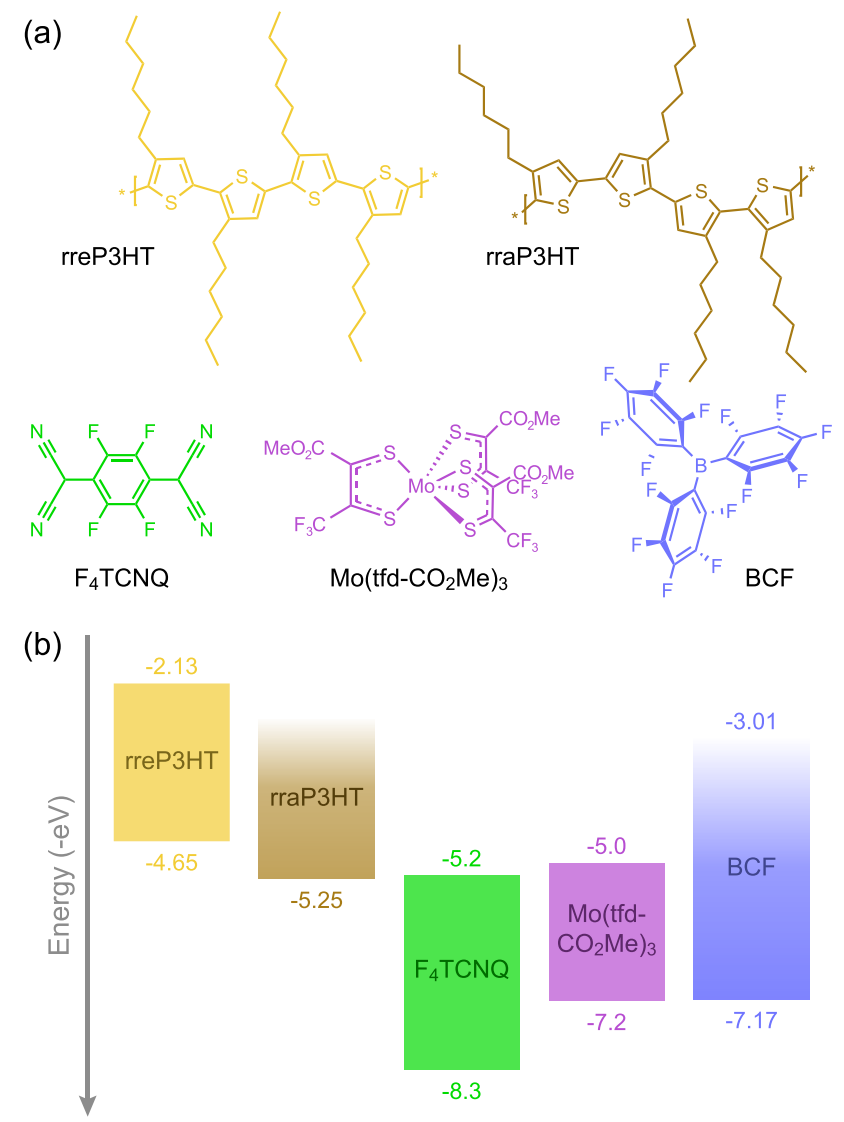

Figure 1. (a) Chemical structures of the polymers and dopants used in this work. (b) HOMO (negative of ionization potential) and LUMO (negative of electron affinity) energy values of the different materials in the solid state (except for the BCF EA, which corresponds to solution data $\left.{ }^{36}\right)$, taken from the literature. ${ }^{33,35,36,40-42}$

proposed to occur on the thiophene ring of the polymer subunit, a similar mechanism can be envisioned for BCF doping of P3HT.

We find that despite the very different chemical nature of the dopants and the different possible doping pathways, all three dopants show similar optical features in rreP3HT, whereas they interact quite differently with raP3HT. We identify three distinctive optical signatures of doped P3HT solutions, investigate the nature of the species contributing to them by EPR, and use ENDOR spectroscopy on flash-frozen solutions to provide direct spectroscopic evidence for the degree of charge delocalization on the P3HT backbone.

\section{EXPERIMENTAL METHODS}

Materials and Sample Preparation. rreP3HT, with $>96 \%$ regioregularity, weight-average molecular weight $\left(M_{\mathrm{w}}\right)$ of ca. $36 \mathrm{kDa}$, and a polydispersity index (PDI) between 2.0 and 2.4 purchased from Rieke Metals, and rraP3HT, with a ca. 1:1 ratio of head-to-head vs head-to-tail couplings, $M_{\mathrm{w}}$ of 37 $\mathrm{kDa}$, and PDI of ca. 2.2 purchased from Sigma-Aldrich, were used as received. The dopants $\mathrm{F}_{4} \mathrm{TCNQ}$ BCF, and bis(trifluoromethanesulfonyl)imide acid (HTFSI) were purchased from TCI Europe and used as received, without further purification. The dopant $\mathrm{Mo}\left(\mathrm{tfd}-\mathrm{CO}_{2} \mathrm{Me}\right)_{3}$ was synthesized according to ref 33 .
If not stated otherwise, the polymer and dopant were dissolved separately in chloroform (CF) and mixed according to the required doping ratios in an inert $\mathrm{N}_{2}$ atmosphere. We used a fairly high concentration of the final solution to ensure that the absorbance of the doping-induced species is above the noise level while at the same time allowing for a fairly small dopant to polymer ratio of $1: 100$. The latter is important to rule out spin-spin interactions along the same polymer chain. The final P3HT concentration in the solutions was $2 \mathrm{~g} \mathrm{~L}^{-1}$ (1.25 $\mathrm{g} \mathrm{L}^{-1}$ in 1:10 $\mathrm{F}_{4}$ TCNQ-doped rraP3HT), corresponding to a molar concentration of $12 \mathrm{mM}$, unless mentioned otherwise. Note that in the absence of the dopant, under the preparation and experimental conditions used in this study (i.e., room temperature solutions in $\mathrm{CF}$, prepared freshly and measured within ca. $24 \mathrm{~h}$ ), absorption spectroscopy did not reveal significant chain aggregation in rreP3HT solution. For all of the doped solutions, the duration between preparation and measurement was optimized for obtaining the strongest and most reliable NIR absorption. In the case of $\mathrm{F}_{4}$ TCNQdoped rreP3HT and $\mathrm{rraP} 3 \mathrm{HT}$, the characteristic optical features appeared immediately after the dopant solution was added to the dissolved polymer and did not increase further with time. Instead, the BCF- and $\mathrm{Mo}\left(\mathrm{tfd}-\mathrm{CO}_{2} \mathrm{Me}\right)_{3}$-doped solutions were stirred slowly for about $24 \mathrm{~h}$ (doped rreP3HT) and $4 \mathrm{~h}$ (doped rraP3HT) in the glovebox (light on) before being measured. The $\mathrm{Mo}\left(\mathrm{tfd}-\mathrm{CO}_{2} \mathrm{Me}\right)_{3}$ anion (for subsequent UV-vis-NIR reference measurements) was generated by adding a few microliters of deionized water to a neutral $\mathrm{Mo}\left(\mathrm{tfd}-\mathrm{CO}_{2} \mathrm{Me}\right)_{3}$ solution $(300 \mu \mathrm{L})$ in anhydrous tetrahydrofuran. ${ }^{34}$ Brønsted-acid (protic) doping was performed by directly adding $1-2$ crystals of HTFSI into $500 \mu \mathrm{L}$ of rre- and rraP3HT solutions in CF. Care was taken to avoid exposure of HTFSI to ambient air by always handling it in the glovebox.

Temperature-dependent measurements were additionally performed in chlorobenzene (CB) solutions in order to be able to access higher temperatures.

UV-vis-NIR Spectroscopy. UV-vis-NIR absorption measurements were carried out in a Varian Cary 5000 spectrophotometer. The solutions were transferred into 1 mm path length precision quartz cuvettes inside the glovebox and subsequently measured outside the glovebox. The background due to solvent contribution was subtracted from the final results. The recorded spectra were analyzed using OriginPro. For the temperature dependence studies, the instrument was fitted with a temperature-controlled cuvette holder TCL 50 by Quantum Northwest. The solutions were maintained at each temperature for $10 \mathrm{~min}$ for equilibration and subsequently measured. Each measurement lasted ca. 7 $\mathrm{min}$, so the total time at each temperature was ca. $17 \mathrm{~min}$. Quantitative analysis of the spectra was done using the BeerLambert law. Additional information about the analysis is given in the SI.

FTIR Spectroscopy. Infrared absorption spectra were recorded in transmission mode with a Bruker Vertex $70 \mathrm{~V}$ FTIR spectrometer at a resolution of $4 \mathrm{~cm}^{-1}$ using 256 scans and an aperture size of $1 \mathrm{~mm}$. Measurements were performed in a liquid transmission cell (Omni-Cell Specac) in which doped P3HT solutions in CF were measured between two silicon windows with a $110 \mu \mathrm{m}$ thick PTFE spacer. A background spectrum of CF measured under the same conditions was subtracted from all data.

EPR and ENDOR Spectroscopy. For the EPR measurements, $25 \mu \mathrm{L}$ of the doped/undoped solution was transferred 
(a)

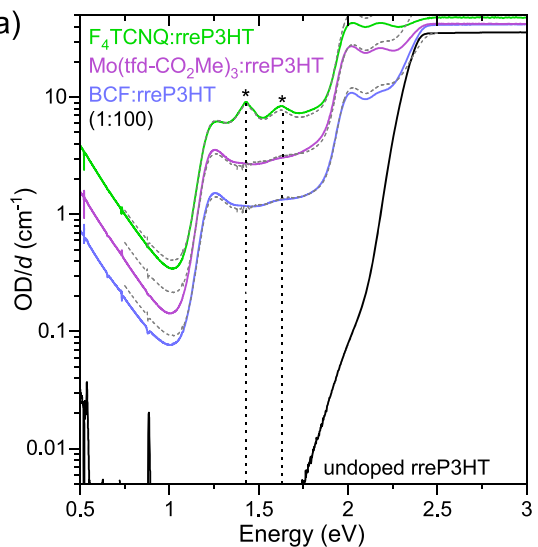

(b)

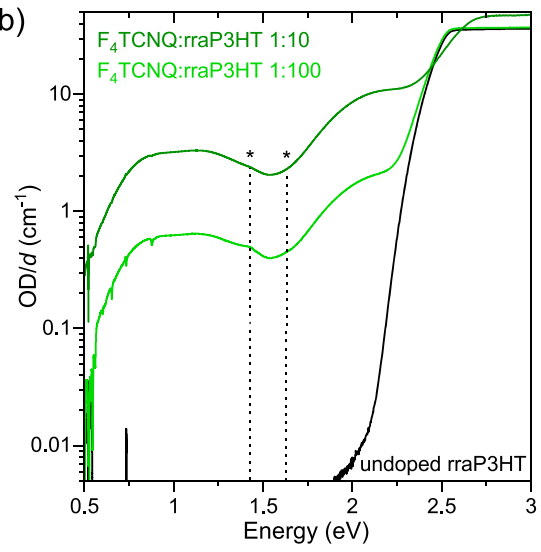

(c)

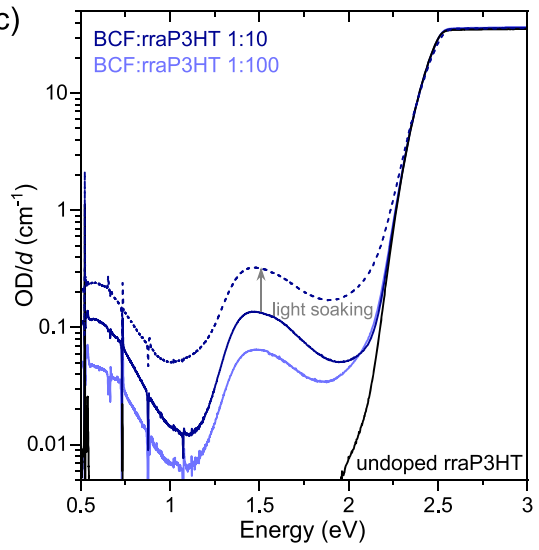

Figure 2. UV-vis-NIR absorption spectra of solutions (in chloroform) of (a) rreP3HT doped with $\mathrm{F}_{4} \mathrm{TCNQ}, \mathrm{Mo}\left(\mathrm{tfd}-\mathrm{CO}_{2} \mathrm{Me}\right)_{3}$ and $\mathrm{BCF}$ in a ratio of 1:100 (dopant: repeat unit), (b) rraP3HT with $\mathrm{F}_{4}$ TCNQ (1:100 and 1:10), and (c) rraP3HT with BCF (1:100, 1:10, light soaked 1:10). Dashed gray lines in (a) correspond to the spectra reconstructed using the Beer-Lambert law with a weighted fraction of the P3HT radical cation (polaron) spectrum (and the $\mathrm{F}_{4}$ TCNQ radical anion spectrum in $\mathrm{F}_{4}$ TCNQ-doped solution). Positions of the characteristic double peaks of the $\mathrm{F}_{4} \mathrm{TCNQ}$ radical anion are highlighted $(*)$ for reference. Additional details are provided in the SI.

into a $2.9 \mathrm{~mm}$ o.d., $2.0 \mathrm{~mm}$ i.d. quartz EPR tube inside a glovebox, and the tube was then sealed with Critoseal. Roomtemperature X-band continuous-wave EPR measurements were performed on a Magnettech MS5000 spectrometer with a liquid-nitrogen-based temperature-control unit. The measurements were performed at a microwave power of $63 \mu \mathrm{W}$ with a modulation frequency of $100 \mathrm{kHz}$ and modulation amplitudes in the range from 0.005 to $0.05 \mathrm{mT}$ depending on the sample. The number of spins was determined from the double integral of the experimental spectrum, taking the experimental settings and the $Q$ factor of the resonator, automatically recorded by the spectrometer software before each scan, into account. ${ }^{43}$ Pulse EPR and Davies ENDOR measurements were performed at Q-band on a Bruker ElexSys E580 spectrometer equipped with a home-built Q-band ENDOR resonator. The samples were flash-frozen in liquid nitrogen, and the measurements were performed at a temperature of $10 \mathrm{~K}$ using a continuousflow helium cryostat and temperature-control system from Oxford Instruments. Further details about the measurement procedure and data evaluation are provided in the SI.

Ab Initio Calculations. Time-dependent density-functional theory (TDDFT) calculations were performed using the package Gaussian 16. ${ }^{44}$ The cc-pVTZ basis set was used in the calculations of protonated oligothiophene, while for the oligothiophene- $\mathrm{F}_{4}$ TCNQ complexes the cc-pVDZ basis set was adopted. The exchange-correlation potential was approximated with the range-separated CAM-B3LYP hybrid functional. ${ }^{45}$ The dispersion interactions were accounted for using the Grimme DFT-D3 scheme. ${ }^{46}$ All structures were relaxed until the residual forces were smaller than $10^{-5} \mathrm{Ha} / \mathrm{bohr}$. The solvent was treated implicitly using the polarizable continuum model $(\mathrm{PCM})^{47}$ with values of the dielectric constants $\varepsilon_{0}=$ 78.36 and $\varepsilon_{\infty}=1.78$ for water and $\varepsilon_{0}=4.71$ and $\varepsilon_{\infty}=2.09$ for chloroform.

\section{RESULTS}

UV-vis-NIR Absorption Spectroscopy. The UV-visNIR spectra of rreP3HT solution doped with $\mathrm{F}_{4}$ TCNQ, $\mathrm{Mo}\left(\mathrm{tfd}-\mathrm{CO}_{2} \mathrm{Me}\right)_{3}$, and BCF in a ratio of $1: 100$, corresponding to one dopant molecule per 100 repeat units of 3hexylthiophene $(3 \mathrm{HT})$, are shown in Figure 2a. This dopant concentration was chosen because of the fairly poor solubility of $\mathrm{F}_{4}$ TCNQ and of doped P3HT. All three systems exhibit the same NIR spectral features, albeit at different absorbances: a low-energy feature extending below $0.5 \mathrm{eV}$ and a broad doublepeaked absorption in the range $1.25-1.75 \mathrm{eV}$. On the basis of previous studies, we assign these to the dopant-induced polaron on the P3HT backbone. Consistent with the literature, the $\mathrm{F}_{4} \mathrm{TCNQ}$-doped rreP3HT spectrum additionally shows the characteristic double peak of the $\mathrm{F}_{4}$ TCNQ radical anion at 1.4 and $1.6 \mathrm{eV}^{3,48}$ In the case of $\mathrm{Mo}\left(\mathrm{tfd}-\mathrm{CO}_{2} \mathrm{Me}\right)_{3}$ doping, the anion absorption peaks are around 1.25 and $2.0 \mathrm{eV}^{34}$ (see Figure S4) but are weak, and hence overshadowed by the signal of the P3HT polaron. As mentioned in our earlier work, there is no appreciable vis-NIR signal from a BCF radical anion, which is expected to occur at about $2.05 \mathrm{eV},{ }^{36,49}$ in the BCF-doped rreP3HT solution. ${ }^{37}$ We finally note that all three spectra display the strong absorption of aggregated P3HT chains around $2 \mathrm{eV}$, which is absent in the undoped P3HT solution and therefore implies doping-induced aggregation. ${ }^{50}$

Since all three doped rreP3HT solutions exhibit ICT features, we were able to analyze the spectra to estimate the ionization efficiency, i.e., the fraction of $\mathrm{P} 3 \mathrm{HT}$ polarons formed per added number of neutral dopants. By making use of the known extinction coefficient of the $\mathrm{F}_{4}$ TCNQ anion ${ }^{48}$ and P3HT polaron (see ref 37 and SI section for details), we found that nearly $66 \%$ of the added $\mathrm{F}_{4}$ TCNQ molecules underwent ionization to form $\mathrm{P} 3 \mathrm{HT}$ polarons and $\mathrm{F}_{4} \mathrm{TCNQ}$ anions, consistent with earlier results. ${ }^{3}$ On the other hand, the P3HT polaron signal in solutions doped with $\mathrm{Mo}(\mathrm{tfd}-$ $\left.\mathrm{CO}_{2} \mathrm{Me}\right)_{3}$ and BCF corresponds to ionization efficiencies of only ca. $35 \%$ and $15 \%$, respectively. Moreover, both BCF and $\mathrm{Mo}\left(\mathrm{tfd}-\mathrm{CO}_{2} \mathrm{Me}\right)_{3}$ do not dope instantly, in contrast to $\mathrm{F}_{4}$ TCNQ (see Figures S2 and S4). This may be a result of steric hindrance between the dopants and the polymer chains ${ }^{30,37}$ but could also be related to the energy level offsets. For example, the lower EA of $\mathrm{Mo}\left(\mathrm{tfd}-\mathrm{CO}_{2} \mathrm{Me}\right)_{3}$ compared to that of $\mathrm{F}_{4} \mathrm{TCNQ}$ implies a smaller driving force for electron transfer from the P3HT HOMO. In the case of BCF doping, a simple one-electron transfer cannot occur and, therefore, additional kinetic barriers are anticipated. We observed that white light illumination increases the doping efficiency in $\mathrm{CF}$ 
(Figure S2), presumably helping overcome or circumvent these barriers. A similar effect has been reported for BCF doping of a hole-transporting polymer poly(triarylamine) (PTAA). ${ }^{51}$ As mentioned earlier, BCF doping of conjugated polymers was proposed to involve formation of a protonated polymer segment in an endothermic process (see below). ${ }^{38}$ Despite the very different chemical nature and shape of the dopants and possibly a different pathway for polaron formation, the shape of the dopant-induced NIR absorption is exactly the same for all three dopant molecules. Since the P3HT polaron spectrum is sensitive to the degree of charge localization and interchain interaction, ${ }^{20,23}$ the insensitivity of the dopinginduced NIR spectra (in rreP3HT) to the choice of the dopant provides first evidence of a very similar nature and environment of the doping-induced species.

The optical spectra of doped rraP3HT reveal significant differences compared to the results obtained with rreP3HT. First, we note that the UV-vis-NIR spectra of Mo(tfd$\left.\mathrm{CO}_{2} \mathrm{Me}\right)_{3}$-doped rraP3HT show no features in addition to the absorption of neutral rraP3HT and the neutral dopant (see Figure S5). This led us to conclude that $\mathrm{Mo}\left(\mathrm{tfd}-\mathrm{CO}_{2} \mathrm{Me}\right)_{3}$ does not dope rraP3HT, which is consistent with the ionization energies and electron affinities shown in Figure $1 \mathrm{~b}$.

The UV-vis-NIR spectra of $\mathrm{F}_{4}$ TCNQ-doped rraP3HT at ratios of $1: 100$ and $1: 10$ in Figure $2 b$ display pronounced subband-gap absorption bands, whose position and shape differ substantially from those in $\mathrm{F}_{4}$ TCNQ-doped rreP3HT. The characteristic $\mathrm{F}_{4} \mathrm{TCNQ}$ anion peaks are almost absent (a faint peak at ca. $1.4 \mathrm{eV}$ is visible in 1:100 $\mathrm{F}_{4}$ TCNQ-doped rraP3HT). Instead, the vis-NIR absorption of $\mathrm{F}_{4}$ TCNQdoped rraP3HT consists of a broad feature between 0.8 and $1.2 \mathrm{eV}$, probably composed of two peaks, and an additional broad peak at ca. $2.0 \mathrm{eV}$. Also, the characteristic peak of P3HT chain aggregation is missing. The absorption spectrum is reminiscent of the film spectrum of $\mathrm{F}_{4} \mathrm{TCNQ}$-doped quaterthiophene $(4 \mathrm{~T})$, which has been attributed to CTC formation. ${ }^{10}$ Similar UV-vis-NIR features (broad absorption bands at around 1.1 and $2.1 \mathrm{eV}$ ) have also been observed in $\mathrm{F}_{4}$ TCNQ-doped rraP3HT films ${ }^{28}$ and P3EHT (a polythiophene-derivative with branched side chains) $)^{29}$ and also assigned to CTCs.

To corroborate this hypothesis and to rationalize the experimental data, we performed a first-principles study on three thiophene oligomers $(\mathrm{T})$ with an increasing number of rings, ranging from four (4T) to eight $(8 \mathrm{~T})$, doped by $\mathrm{F}_{4}$ TCNQ. We consider different lengths of the oligothiophenes to account for the random size of the regular regions in rraP3HT. The systems are modeled as bimolecular complexes, as in previous work, ${ }^{52,53}$ but in this case, we additionally consider the embedding in an implicit solvent (chloroform) to mimic the experimental conditions more realistically.

The calculated optical absorption spectrum of $6 \mathrm{~T}-\mathrm{F}_{4} \mathrm{TCNQ}$ shown in Figure 3a, is characterized by three dominant peaks: $E_{1}$ corresponds to the transition from the bonding HOMO to the antibonding LUMO of the CTC, ${ }^{52}$ while $E_{2}$ is related to the transition from the HOMO-1 to the LUMO of the complex. Increasing the oligothiophene length does not affect the character of $E_{1}$, while it makes $E_{2}$ more charge-transfer-like, due to the increasing localization of the HOMO-1 on the donor. $^{52}$ On the other hand, the intense peak $E_{3}$, originating from intramolecular transitions, is rather sensitive to the donor length. ${ }^{52}$ The spectrum obtained in vacuo closely resembles those presented in refs 52 and 53. Minor differences are due to (a)

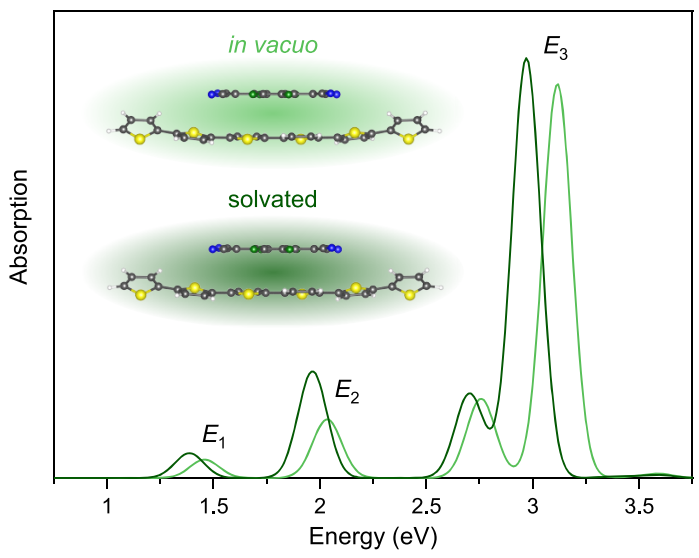

(b)

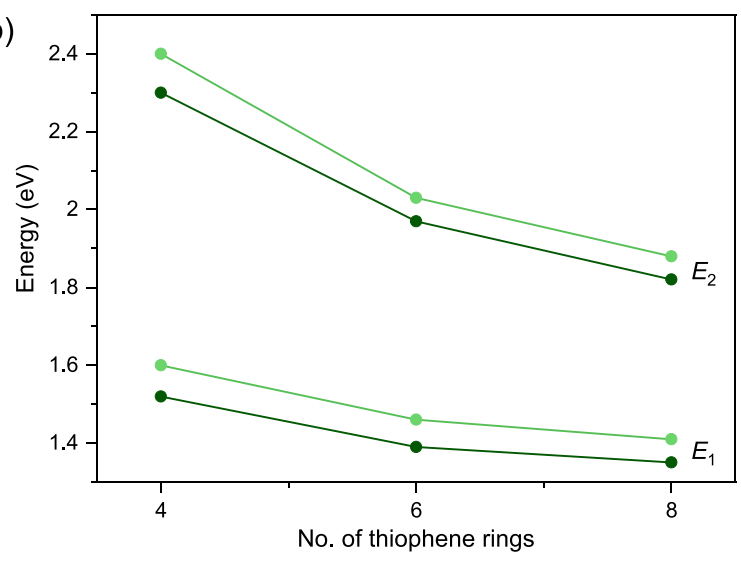

Figure 3. (a) Optical absorption spectra from TDDFT of the 6T:F4TCNQ bimolecular complex in vacuo (light green) and in chloroform (dark green). (Insets) Respective optimized geometries. (b) Energy of the absorption peaks $E_{1}$ and $E_{2}$ as a function of the length of the doped oligothiophene in vacuo (light green) and in chloroform (dark green). Lorentzian broadening of $80 \mathrm{meV}$ is applied to all spectra.

the different computational parameters adopted in the two sets of calculations, which affect the initial geometry of the system. In solution, the relaxed bimolecular structure changes slightly compared to the configuration in vacuo, resulting in a reduced upward bend of the outer thiophene rings of the oligomer (see insets of Figure 3a). Consequently, the amount of charge transfer in the ground state, calculated from the Mulliken charge analysis, is enhanced from 0.24 in vacuo to 0.29 in solution, consistent with similar results reported in the literature. ${ }^{54} \mathrm{We}$ emphasize that these values are obtained for the isolated molecular pairs. We checked that the presence of alkyl chains increases the amount of charge transfer, leading to results in better agreement with experiments. We also recall that the charge-transfer values computed from DFT are highly sensitive to the approximation of the exchange-correlation potential as well as to the adopted partition scheme. Differences of this kind explain the discrepancy between the results obtained here and those reported in the literature on the same systems in vacuo. ${ }^{10,53}$ The main effect of the solvent is to red-shift the spectrum almost rigidly by about $100 \mathrm{meV}$ (see Figure 3a). The same behavior is exhibited by the bimolecular complexes with all considered thiophene oligomers (see Figure $3 b$ ), demonstrating that the effect of solvation is independent of the length of the thiophene chain. 
Now, extrapolating the trend shown in Figure $3 \mathrm{~b}$ toward infinite chain lengths, it is clear that the absorption onset will not drop below $1 \mathrm{eV}$, as observed in the measurement (Figure 2b). We explain this behavior as due to the interactions between neighboring chains, not included in our model, which enhance the overall screening and thus red shift the spectrum by a few hundred millielectronvolts compared to the isolated system. $^{55-57}$

Assuming that these interactions do not change the character of the excitations, we assign the plateau between 0.5 and $1.6 \mathrm{eV}$ in the experimental spectra (Figure $2 \mathrm{~b}$ ) to peaks $E_{1}$ and $E_{2}$, i.e., to excitations with hybrid- and charge-transfer character. Moreover, we associate the increased absorption between 1.6 and $2.2 \mathrm{eV}$ with $E_{3}$, i.e., with intramolecular/ intrapolymeric excitations locally altered with respect to the undoped material due to formation of CTCs.

Next, we turn to the UV-vis-NIR spectra of BCF-doped rraP3HT solutions, shown in Figure 2c. The broad absorption seen between 1.25 and $1.75 \mathrm{eV}$ for its regioregular counterpart is replaced by a single peak at $1.5 \mathrm{eV}$, and the low-energy absorption maximum blue-shifts to $0.6 \mathrm{eV}$. Again, there is no evidence for aggregation of the neutral polymer sections at ca. $2 \mathrm{eV}$. In addition, the doping-induced signal is rather weak for both the 1:100 and the 1:10 solution, indicative of either a low concentration of doped polymer segments or formation of a species with a very low oscillator strength. We were, however, able to increase the signal by a factor of 2 by light soaking/ illumination as with BCF-doped rreP3HT solutions. As pointed out above, similar absorption features have been reported before and were assigned to polarons located in amorphous P3HT domains or individual chains. ${ }^{12,24,25}$ We had shown in an earlier publication that the pronounced doublepeaked polaron absorption in the NIR region of BCF-doped rreP3HT solutions in $\mathrm{CB}$ transforms into a broad peak centered around $1.5 \mathrm{eV}$ upon heating beyond $70{ }^{\circ} \mathrm{C} .{ }^{37}$ This was accompanied by the suppression of the $\mathrm{P} 3 \mathrm{HT}$ aggregate peak at $2 \mathrm{eV}$, which suggests a more localized $\mathrm{P} 3 \mathrm{HT}$ polaron on individual nonaggregated chains as the origin of the characteristic NIR absorption we see in BCF-doped rraP3HT. This assignment was recently confirmed by absorption experiments on nonfiltered and filtered solutions of BCFdoped rreP3HT. ${ }^{19}$ Again, none of the spectra reveal additional peaks in the spectral range considered here, which is expected if the counterion is the $\mathrm{BCF}-\mathrm{OH}^{-}$anion or a similar species, since these species are expected to absorb at higher energy.

Summarizing the results from optical spectroscopy, doping of rreP3HT with all three dopants induces the very same NIR absorption. This implies that the same polaron species (a delocalized polaron on planarized/aggregated chains) is formed on the polymer backbone. Importantly, the shape and spectral position of the doping-induced spectrum is independent of (1) the chemical structure of the dopant, (2) the nature of the doping process, and (3) the interaction of the dopant-derived anion with the positively charged polymer backbone. In contrast, the absorption spectrum of doped rraP3HT is strongly dependent on the dopant, suggesting formation of a CTC state and a more localized polaron on isolated polymer chains in the case of $\mathrm{F}_{4} \mathrm{TCNQ}$ and BCF, respectively. Except for the anion of $\mathrm{F}_{4} \mathrm{TCNQ}$ the absorption spectra do not reveal any additional information on the existence and nature of other doping-induced species. This motivated a comprehensive study of the doped polymer solutions using EPR spectroscopy.
EPR Spectroscopy. Doping via ICT typically leads to formation of species with unpaired electrons in their ground state that give an EPR signal, whereas CTCs and bipolarons are diamagnetic and, therefore, EPR silent. By quantifying the amount of charge carriers with unpaired electron spins using EPR and comparing the results to the UV-vis-NIR measurements, the assignments of the observed absorption bands can be confirmed, and the ICT and CTC mechanisms can be distinguished. Moreover, the EPR spectral signatures are determined by the molecular environment of the unpaired electron, and precise information on the interaction of the unpaired electron with surrounding magnetic nuclei can be extracted using pulse EPR techniques, thereby providing insight into the chemical nature of the paramagnetic species formed during the doping process. Due to the abovementioned influence of time and/or illumination on the ionization efficiency in some of the doped solutions, UV-visNIR and EPR measurements were typically performed in parallel using the same stock solutions.

The room-temperature continuous wave $(\mathrm{cw}) \mathrm{X}$-band EPR spectra recorded on rreP3HT solutions doped with $\mathrm{F}_{4} \mathrm{TCNQ}$ $\mathrm{Mo}\left(\mathrm{tfd}-\mathrm{CO}_{2} \mathrm{Me}\right)_{3}$, and BCF are compared in Figure 4a. Doping of rreP3HT with both $\mathrm{Mo}\left(\mathrm{tfd}-\mathrm{CO}_{2} \mathrm{Me}\right)_{3}$ and BCF leads to an EPR signal at $g=2.0022$, assigned to the radical cation on the P3HT backbone in agreement with the literature. ${ }^{58}$ In the case of $\mathrm{Mo}\left(\mathrm{tfd}-\mathrm{CO}_{2} \mathrm{Me}\right)_{3}$-doped rreP3HT, an additional signal with equal integrated intensity is present at $g=2.0085$ and is assigned to the dopant radical anion based on comparison to literature $g$ values of similar monoanionic molybdenum complexes. ${ }^{59,80}$ The EPR spectrum of $\mathrm{F}_{4} \mathrm{TCNQ}$ doped rreP3HT shows a signal at a slightly larger $g$ value of 2.0025 and an additional superimposed signal with resolved hyperfine structure. The latter was assigned to free $\mathrm{F}_{4} \mathrm{TCNQ}$ anions in solution with a $g$ value of 2.0030 and hyperfine couplings of 3.1 and $4.4 \mathrm{MHz}$ to the four equivalent nitrogen and the four equivalent fluorine nuclei, respectively, based on additional measurements on the $\mathrm{F}_{4} \mathrm{TCNQ}$ radical anion generated in THF solution ${ }^{3}$ (see SI and Figure S7 for details). Simulations reveal that this contribution of free $\mathrm{F}_{4} \mathrm{TCNQ}$ radical anions amounts to only $1 \%$ of the spins present in solution (see Figure S8). The larger contribution is given by the signal at an intermediate $g$ value with respect to the P3HT and $\mathrm{F}_{4} \mathrm{TCNQ}$ radicals. Early EPR studies on TCNQ chargetransfer salts have interpreted similar observations in terms of exchange interactions between the ions with $|J| \gg\left(g_{1}-\right.$ $\left.g_{2}\right) \mu_{\mathrm{B}} \mathbf{B}$, where $|J|$ is the exchange coupling, $\left(g_{1}-g_{2}\right)$ is the difference in $g$ values between the two radical species, $\mu_{\mathrm{B}}$ is the Bohr magneton, and $\mathbf{B}$ is the applied static magnetic field. ${ }^{61-63}$ We therefore assign the observed signal to exchange-coupled $\mathrm{F}_{4}$ TCNQ radical anions and $\mathrm{P} 3 \mathrm{HT}$ radical cations within the P3HT aggregates formed in solution upon doping. The close proximity of the dopant and the charge on $\mathrm{P} 3 \mathrm{HT}$ after doping suggested by this is in accordance with the observation of low carrier mobilities in $\mathrm{F}_{4}$ TCNQ-doped P3HT, despite observation of a significant ionization efficiency. ${ }^{3}$ For $\mathrm{Mo}(\mathrm{tfd}-$ $\left.\mathrm{CO}_{2} \mathrm{Me}\right)_{3}$-doped $\mathrm{P} 3 \mathrm{HT}$, the observation of individual signals from the P3HT radical cation and dopant anion and the absence of broadening compared to the BCF-doped P3HT spectrum indicate that, if present, the exchange coupling is not sufficiently strong to affect the X-band EPR spectrum.

For BCF-doped P3HT, the EPR measurements revealed the presence of only the free radical cation on the P3HT backbone. This assignment was unambiguously confirmed by 
(a)

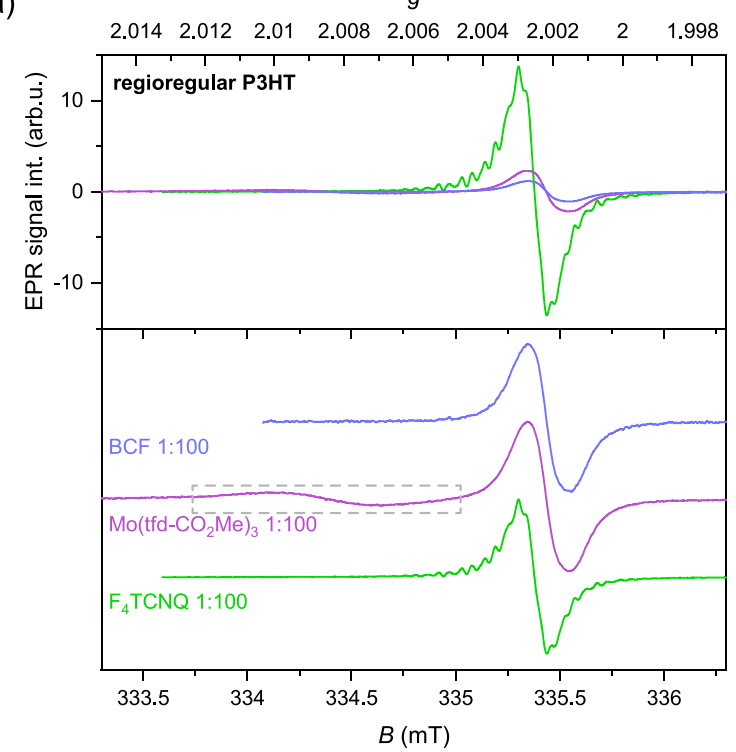

(b)

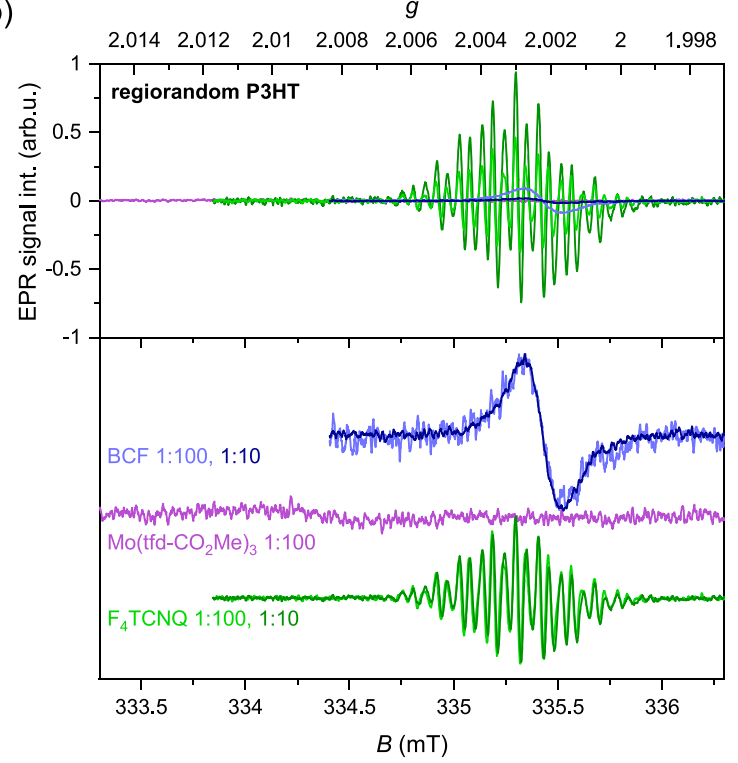

Figure 4. X-Band room-temperature cw EPR spectra recorded on solutions of rreP3HT (a) and $\operatorname{rraP} 3 \mathrm{HT}$ (b) doped with $\mathrm{F}_{4} \mathrm{TCNQ}$ $\mathrm{BCF}$, or $\mathrm{Mo}\left(\mathrm{tfd}-\mathrm{CO}_{2} \mathrm{Me}\right)_{3}$ in chloroform at a doping ratio of $1: 100$ (and 1:10 for rraP3HT). Signal intensities normalized by the $Q$ factor and modulation amplitude are compared in the top part of the graphs, and normalized spectra are shown in the bottom part for comparison of the spectral shapes. The signal from the $\mathrm{Mo}\left(\mathrm{tfd}-\mathrm{CO}_{2} \mathrm{Me}\right)_{3}$ anion is highlighted by the dashed gray box. Spectra are shown for a microwave frequency of $9.4 \mathrm{GHz}$; additional experimental details are described in the Experimental Methods and the SI.

additional W-band EPR measurements on a flash-frozen solution of BCF-doped P3HT that allowed resolution of the rhombic $g$ tensor and showed excellent agreement with previous results on $\mathrm{I}_{2}$-doped P3HT (see Figure S10). ${ }^{58}$ The presence of only the $\mathrm{P} 3 \mathrm{HT}$ radical cation is inconsistent with the proposed mechanism for the BCF doping of PCPDTBT, ${ }^{38}$ in which a second radical species, the neutral protonated P3HT chain, is formed, while $\mathrm{BCF}-\mathrm{OH}^{-}$is spinless and EPR silent. To further investigate the possibility that BCF doping of $\mathrm{P} 3 \mathrm{HT}$ proceeds by chain protonation, we complemented our studies with UV-vis-NIR and EPR experiments on rre- and rraP3HT solutions doped with the Brønsted acid dopant HTFSI. ${ }^{29,64}$ Figure $5 \mathrm{a}$ and $5 \mathrm{~b}$ shows the UV-vis-NIR and EPR spectra of the HTFSI-doped P3HT solutions in comparison to BCF-doped solutions, respectively. The proposed reaction mechanism for protonic acid doping of P3HT is shown in Figure 5c. The UV-vis-NIR spectra of BCF- and HTFSI-doped rreP3HT solutions are found to be nearly identical with respect to spectral shape, both showing the characteristic ICT polaronic features (low-energy transition with a maximum below $0.5 \mathrm{eV}$, a broad shoulder at 1.25-1.75 $\mathrm{eV}$, and an aggregate absorption at $2 \mathrm{eV}$ ). Moreover, the $\mathrm{cw}$ EPR spectra of HTFSI-doped solutions were found to be identical to the spectra recorded for BCF-doped P3HT, as shown in Figure 5b (see also Figure S10), indicating, in both cases, formation of P3HT radical cations as well as the absence of a second EPR signature attributable to another radical species such as the neutral radical on the protonated chain. The difference in the spin density distribution in the protonated neutral P3HT radical compared to the radical cation would be expected to lead to a change both in $g$ values and in the extent of spin delocalization determined by ENDOR (vide infra), neither of which are observed experimentally. However, these findings are consistent with an overall reaction

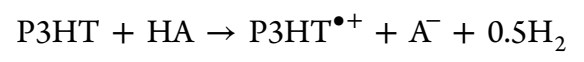

where $\mathrm{HA}=\mathrm{BCF} \cdot \mathrm{H}_{2} \mathrm{O}$ or HTFSI, similar to what has been suggested for the reaction of ferrocene and $\mathrm{BCF} \cdot \mathrm{OH}_{2}{ }^{39}$ It may well be that the reaction proceeds in a similar fashion to that suggested for PCPDTBT but that two neutral protonated radical species subsequently react together to eliminate hydrogen and regenerate neutral closed-shell P3HT. Alternatively, two protonated P3HT cations could react to give two polarons and $\mathrm{H}_{2}$ or a protonated cation and a protonated radical could react to give a polaron, neutral $\mathrm{P} 3 \mathrm{HT}$, and $\mathrm{H}_{2}$.

In Table 1, we compare the efficiency of rreP3HT polaron generation per dopant molecule determined from analysis of the NIR absorption spectra and from the spin concentrations obtained by EPR. Good agreement is found assuming that BCF creates one spin per ICT, while doping with $\mathrm{F}_{4} \mathrm{TCNQ}$ and $\mathrm{Mo}\left(\mathrm{tfdCO}_{2} \mathrm{Me}\right)_{3}$ via ICT yields two radicals (the dopant radical anion and the $\mathrm{P} 3 \mathrm{HT}$ radical cation), consistent with the EPR spectral assignments. Importantly, the close agreement between the doping efficiencies from optical spectroscopy and EPR rules out a significant contribution from bipolarons to the NIR region of the optical spectrum. With this information in hand, we recalculated the molar extinction spectrum of polarons on aggregated rreP3HT by now taking into consideration the amount of charged $\mathrm{P} 3 \mathrm{HT}$ present in the system measured by EPR to obtain a more accurate estimate, as shown in Figure 6a.

Similar to the results of the UV-vis-NIR measurements, the EPR measurements on rraP3HT show significant differences compared to rreP3HT. The EPR signals observed in doped rraP3HT solutions shown in Figure $4 \mathrm{~b}$ are significantly weaker than in the corresponding rreP3HT solutions for the same doping ratio (1:100). In the case of $\mathrm{Mo}\left(\mathrm{tfd}-\mathrm{CO}_{2} \mathrm{Me}\right)_{3}$ doped rraP3HT, the signal in solution seems to consist almost exclusively of the signal of a small quantity of the paramagnetic dopant anion at $g=2.0085$ (presumably formed by adventitious reduction by water), in agreement with the UV-vis-NIR results. The presence of some doped rraP3HT 
(a)

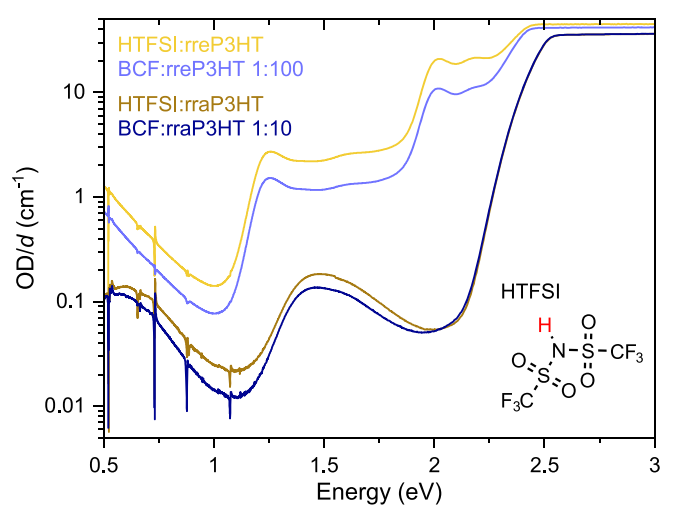

(b)

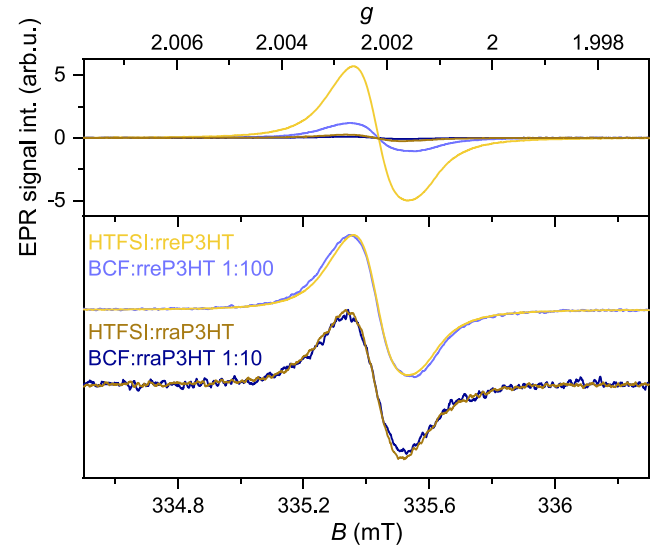

(c)
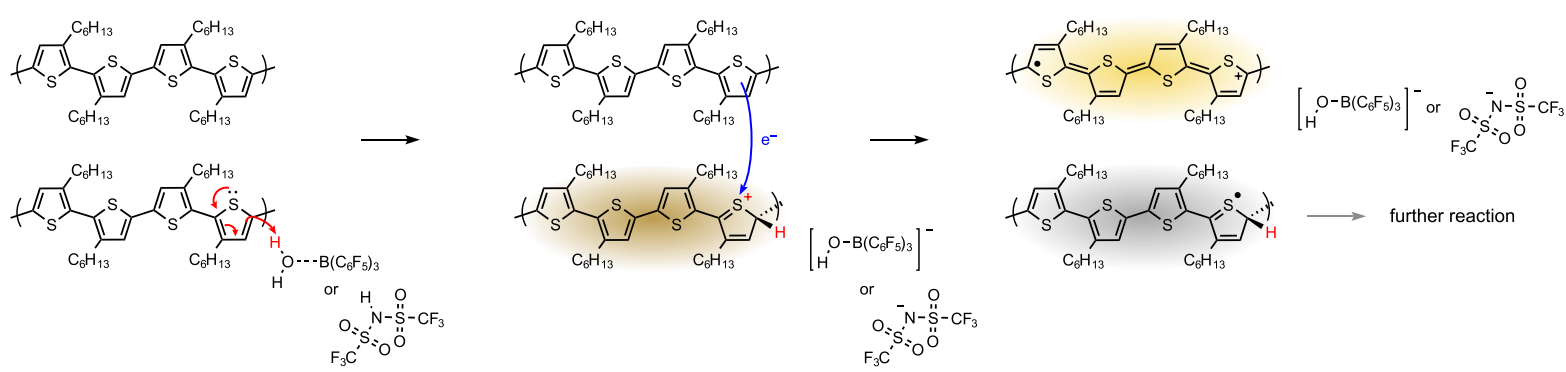

Figure 5. (a) Comparison of UV-vis-NIR absorption spectra of rre- and rraP3HT solutions (in chloroform) doped with BCF and HTFSI. (Inset) Chemical structure of HTFSI. (b) Comparison of X-band room-temperature cw EPR spectra recorded on solutions of rreP3HT and rraP3HT doped with BCF or HTFSI. Signal intensities normalized by the $Q$ factor and modulation amplitude are compared in the top part of the graphs, and normalized spectra are shown in the bottom part for comparison of the spectral shapes. See the Experimental Methods and the SI for additional experimental details. (c) Possible reaction mechanism between P3HT units and BCF or HTFSI, following refs 38 and 65 , wherein a P3HT chain is first protonated, leading to the creation of a spinless protonated polymer cation (brown) and a negatively charged $\mathrm{BCF}^{-} \mathrm{OH}^{-}$complex. Subsequent electron transfer from a neutral chain or segment results in a P3HT radical cation (golden) and a neutral protonated radical (gray) that is converted to diamagnetic products in further reaction steps (see text for details).

Table 1. Fraction of Doped P3HT per Added Dopant Molecule Estimated from UV-vis-NIR and cw EPR Spectroscopies

\begin{tabular}{|c|c|c|c|c|}
\hline \multirow[b]{2}{*}{ dopant } & \multicolumn{2}{|c|}{ doped rreP3HT per dopant molecule } & \multicolumn{2}{|c|}{ doped rraP3HT per dopant molecule } \\
\hline & UV-vis-NIR, $1: 100^{a}$ & EPR, $1: 100^{b}$ & EPR, $1: 100^{b}$ & EPR, $1: 10^{b}$ \\
\hline $\mathrm{F}_{4} \mathrm{TCNQ}$ & 0.66 & $0.54 \pm 0.05$ & $0.0044 \pm 0.0022$ & $0.0012 \pm 0.0003$ \\
\hline $\mathrm{BCF}$ & 0.15 & $0.12 \pm 0.01$ & $0.0023 \pm 0.0005$ & $0.0008 \pm 0.0001$ \\
\hline $\mathrm{Mo}\left(\mathrm{tfd}-\mathrm{CO}_{2} \mathrm{Me}\right)_{3}$ & 0.35 & $0.27 \pm 0.03$ & $0.0002 \pm 0.0002$ & \\
\hline
\end{tabular}

${ }^{a}$ Estimated from the UV-vis-NIR absorption spectra in Figure 2a (see SI section 1 for details). ${ }^{b}$ Calculated from the number of spins on P3HT determined by quantitative EPR from the spectra in Figure $4 \mathrm{a}$ and $4 \mathrm{~b}$ by considering two spins generated per dopant for $\mathrm{F}_{4} \mathrm{TCNQ}$ and $\mathrm{Mo}$ (tfd$\left.\mathrm{CO}_{2} \mathrm{Me}\right)_{3}$ and one spin per dopant for BCF-doped solutions. The reported error estimate is calculated as the standard deviation from individual measurements.

cannot be ruled out completely, but its concentration would be significantly lower than that of the dopant anion.

The EPR spectrum obtained for $\mathrm{F}_{4} \mathrm{TCNQ}$-doped rraP3HT can be decomposed into a contribution of the free $\mathrm{F}_{4} \mathrm{TCNQ}$ anion in solution, clearly showing a hyperfine structure attributable to coupling to four nitrogen and four fluorine nuclei and a contribution assigned to the radical cation on rraP3HT (see Figure S8) characterized by the same spectrum as that observed in the case of BCF-doped rraP3HT (vide infra). The two species contribute equally to the spectrum and are therefore due to ICT. The corresponding spin concentrations are, however, orders of magnitude lower compared to doped rreP3HT (see Table 1), despite the rather large signal strength in the NIR absorption spectra. This is consistent with the assignment of the absorption features that dominate the NIR spectrum to a CTC, which would be EPR silent.
To further validate this, we conducted FTIR spectroscopy on the $\mathrm{F}_{4} \mathrm{TCNQ}$-doped solutions. When $\mathrm{F}_{4} \mathrm{TCNQ}$ is ionized, the IR bands in the $\mathrm{C} \equiv \mathrm{N}$ stretching region undergo a red-shift, where the magnitude of the shift is proportional to the degree of charge transfer $\delta .{ }^{66}$ In $\mathrm{F}_{4}$ TCNQ-doped rreP3HT, the predominant peak is at ca. $2195 \mathrm{~cm}^{-1}$ as shown in Figure S6 (vs $2227 \mathrm{~cm}^{-1}$ for neutral $\mathrm{F}_{4} \mathrm{TCNQ}^{10,66}$ ). This peak is widely assigned to the $\mathrm{F}_{4} \mathrm{TCNQ}$ anion, suggesting ICT with $\delta=$ 1..$^{10,67}$ In $\mathrm{F}_{4} \mathrm{TCNQ}$-doped rraP3HT on the other hand, the primary peak is centered at ca. $2210 \mathrm{~cm}^{-1}$, corresponding to a degree of charge transfer $\delta$ of only 0.53 (see SI for details). This supports the assignment of the UV-vis-NIR features in Figure $2 \mathrm{~b}$ to a CTC. ${ }^{10,27}$ In addition, the FTIR spectrum of the $\mathrm{F}_{4} \mathrm{TCNQ}$-doped rraP3HT solution displays a small peak near $2195 \mathrm{~cm}^{-1}$, indicating that a fraction of ICT species is also present, though at a much lower concentration. This is consistent with the weak anion absorption peak in the NIR 

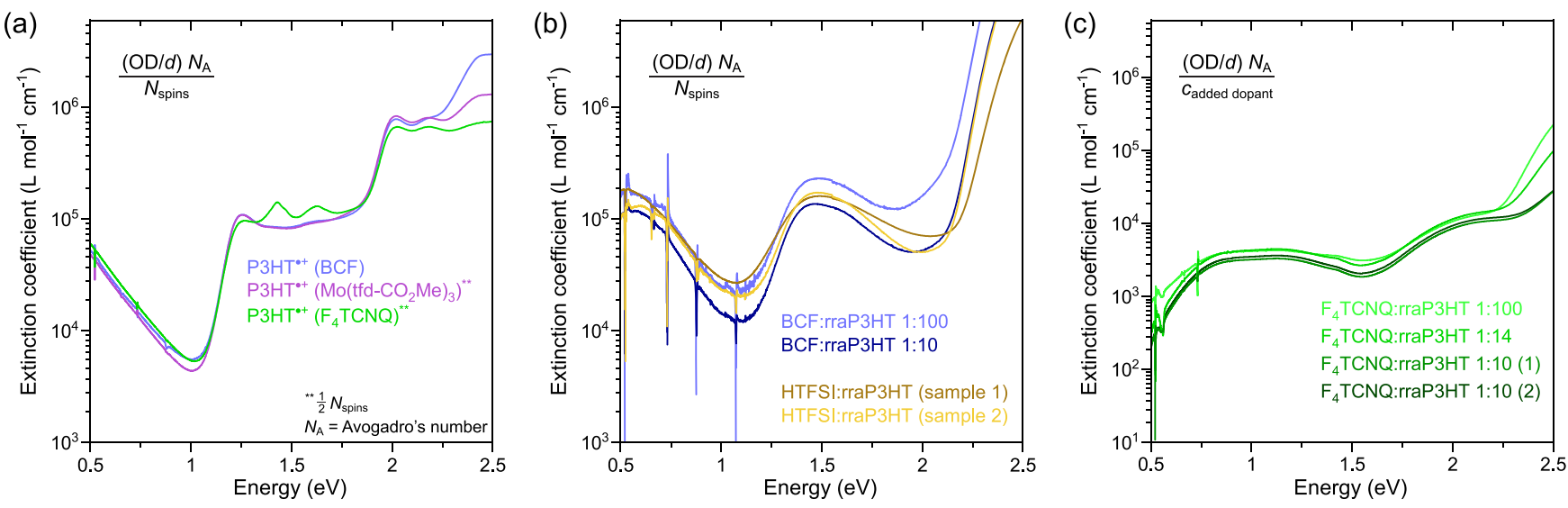

Figure 6. Molar extinction coefficient of a polaron on (a) rreP3HT and (b) rraP3HT, determined using the concentration of spins in doped $\mathrm{P} 3 \mathrm{HT}$ (see Table S2) obtained from EPR spectroscopy. (c) Molar extinction spectrum of the CTC formed between $\mathrm{F}_{4} \mathrm{TCNQ}$ and rraP3HT, calculated from the optical absorption of different data sets with the presumption that every dopant forms one complex. Note that above $1.7 \mathrm{eV}$ the absorption becomes dominated by neutral, aggregated, and nonaggregated P3HT chains, which will contribute differently to the spectra depending on the polymer concentration and the degree of aggregation.

spectrum in Figure $2 b$ (particularly in the 1:100 ratio solution) and with the observation of a small fraction of ICT by EPR in Figure $4 \mathrm{~b}$ (with an efficiency of less than $1 \%$, see Table 1 ). Neelamraju et al. $^{28}$ showed that rraP3HT films exposed to small concentrations of $\mathrm{F}_{4} \mathrm{TCNQ}$ revealed absorption features characteristic of ICT, whereas at higher $\mathrm{F}_{4}$ TCNQ concentrations the optical spectra were dominated by a broad signal attributed to CTC. This finding was interpreted in terms of the broad density of state (DOS) distribution of the highly disordered rraP3HT layer, where only a small fraction of the conjugated neutral segments has a sufficiently low ionization potential (IP) to enable electron transfer to the LUMO of $\mathrm{F}_{4}$ TCNQ. Our rraP3HT has a probability for head-to-tail coupling of approximately $50 \%$, which would still allow a small fraction of sufficiently long head-to-tail-coupled segments suitable for ICT. However, as the FTIR spectrum is dominated by the peak centered at $2210 \mathrm{~cm}^{-1}$ (in particular for the 10:1 doping ratio), we believe that the CTC is the dominant species. Similarly, we also observe a small CTC peak in the FTIR spectrum of $\mathrm{F}_{4}$ TCNQ-doped rreP3HT solutions (Figure S6), implying the coexistence of ICT and CTC states in the doped samples, in concurrence with the results of Neelamraju et al. $^{28}$ The characteristic CTC bands of $F_{4}$ TCNQ-doped P3HT were also observed during temperature-dependent UVvis-NIR/EPR measurements of the doped rreP3HT solutions in CB (see SI for details and discussion).

On the basis of this evidence for assignment of the NIR absorption features to a CTC and ruling out a significant contribution of ionized and neutral $\mathrm{F}_{4}$ TCNQ we calculated the molar extinction spectrum of the CTC with the result shown in Figure 6c. As expected, the extinction coefficient of the CTC is much smaller when compared to that of the polaron on an aggregated rreP3HT chain.

The BCF-doped rraP3HT solutions show an EPR signal at a similar $g$ value as for rreP3HT, which is attributed again to the $\mathrm{P} 3 \mathrm{HT}$ radical cation. However, the overall magnitude of the signal is much smaller compared to the BCF-doped rreP3HT. Notably, EPR measurements on the HTFSI-doped rre- and rraP3HT solutions reveal a situation similar to that observed with BCF-doped P3HT with a spin count almost 2 orders of magnitude higher in HTFSI-rreP3HT $\left(8.7 \pm 0.1 \times 10^{14}\right.$ in 25 $\mu \mathrm{L}$ of solution) compared to HTFSI-rraP3HT $(1.6 \pm 0.5 \times$ $10^{13}$ in $25 \mu \mathrm{L}$ of solution). The HTFSI-doped rraP3HT solutions also display absorption features which are very similar in shape and spectral position to those in BCF-doped rraP3HT (e.g., the NIR signal in HTFSI-doped rraP3HT was also influenced by time ${ }^{52}$ and illumination (see Figure S3)). Again, the observation of only one EPR signal rules out the presence of a second radical species (besides the polymer radical cation).

The low EPR signal strength for the BCF-doped rraP3HT solution is consistent with the low absorption strength of the NIR feature and indicates a low concentration of dopinginduced P3HT polarons. If we associate each polymer polaron to formation of one spin, the calculated molar extinction coefficient spectrum of the spin-carrying moiety exhibits a reasonably high absorption strength, comparable to that of the doped rreP3HT samples (see Figure $6 \mathrm{~b}$ ). This supports the assignment of the below-band-gap absorption feature in BCFdoped rraP3HT to a polaron on rraP3HT. The differences in the strength and shape of the spectra of the different samples in Figure $6 \mathrm{~b}$ may in part be due to the to the low absolute absorbance of the 1:100 BCF-doped solution which causes an uncertainty in the background correction but could also indicate differences in chain conformation/interactions depending on the concentration and nature of the dopinginduced species. For example, only the segments with longrange order presumably undergo ionization at low doping concentrations, whereas also the shorter and more disordered chain segments get ionized at higher doping concentrations, hence resulting in a broader distribution of chain lengths in the latter case.

We note, however, that there is some evidence for the coexistence of spinless doping-induced species, particularly in BCF/HTFSI-doped rraP3HT solutions under extended illumination (see Figure $2 \mathrm{c}$ and SI Figure S3 and section 4.4). In order to ascertain that the (also spinless) protonated P3HT cation, which is an intermediate product in the doping reaction (see Figure 5c), does not contribute significantly to the UV-vis-NIR spectrum in Figure $6 \mathrm{~b}$, we turned to DFT. The results of TDDFT calculations on (protonated) 4T doped by $\mathrm{BCF}$ in solution indicate that the main effect of protonation 
is to distort the molecular backbone of the donor or, depending on the site, even to break the conjugation (Figures S11-S13). These mechanisms lead to a segregation of the frontier orbitals and to a consequent splitting of the first absorption peak into two excitations (one of them is dark or very weak) at lower and higher energies with respect to the onset of the neutral species (details and further analysis are in the Supporting Information, section 5). Notably, none of the considered scenarios explains the appearance of a strong absorption well below the absorption onset of the neutral species (Table S3). As such, our TDDFT calculations rule out a significant contribution from protonated chains to the subband-gap absorption in the BCF-doped thiophenes. We conclude that prolonged illumination of BCF/HTFSI-doped rraP3HT causes byproducts of yet unknown origin.

ENDOR Spectroscopy. The above results show distinct differences in the optical absorption of doping-induced species on rreP3HT and rraP3HT. In particular, the observation of a pronounced aggregate absorption peak at $2.0 \mathrm{eV}$ and the redshifted $\mathrm{P}_{1}$ transition of doped rreP3HT solutions in chloroform suggests formation of highly delocalized polarons. As mentioned earlier, various groups proposed the importance of charge delocalization on the mechanism and efficiency of charge transfer. ${ }^{8}$ However, experimental evidence for this conclusion is limited. ${ }^{68}$ Here, we used the pulse EPR technique electron nuclear double-resonance (ENDOR) spectroscopy to estimate the extent of spin delocalization of the $\mathrm{P} 3 \mathrm{HT}$ radical cation for the different combinations of polymer host and dopant. The results of Q-band ${ }^{1} \mathrm{H}$ Davies ENDOR measurements performed on flash-frozen solutions of doped rre- and rraP3HT (in CF) are compared in Figure $7 \mathrm{a}$ and $7 \mathrm{~b}$, respectively.

ENDOR measures the strength of the hyperfine coupling between the unpaired electron spin and the magnetic nuclei, in this case the protons on the polymer backbone. Each proton contributes to the ENDOR spectrum with two lines centered at the nuclear Larmor frequency $\left(\nu_{1 \mathrm{H}}\right)$ and split by the hyperfine coupling. Therefore, contributions near the Larmor frequency correspond to weakly coupled nuclei, while the outer edges of the ENDOR spectra are determined by those protons most strongly interacting with the unpaired electron. In P3HT, the ENDOR spectrum is mainly due to the $\alpha$ protons on the thiophene rings and the corresponding hyperfine couplings are directly proportional to the spin density on the adjacent carbon, therefore allowing determination of the spin density distribution on the polymer backbone. ${ }^{58,69,70}$ Since the spin density is distributed over multiple thiophene rings, several protons with a range of different hyperfine coupling strengths contribute to give an ENDOR spectrum without clear features that could be assigned to individual protons. Nevertheless, the width of the spectrum is still determined by the largest proton hyperfine coupling and scales with the number of thiophene rings over which the electron is delocalized. This leads to a narrow ENDOR spectrum in the case of extensive delocalization and to a broader spectrum in the case of localization of the unpaired electron on a shorter segment of the polymer chain. ${ }^{58,69,70}$

The ENDOR spectra recorded for frozen rreP3HT solutions with the four different dopants have an almost identical width, indicating a similar extent of delocalization. This is in line with the identical shape and spectral position of the polaron absorption spectra with the different dopants, shown in Figures (a)

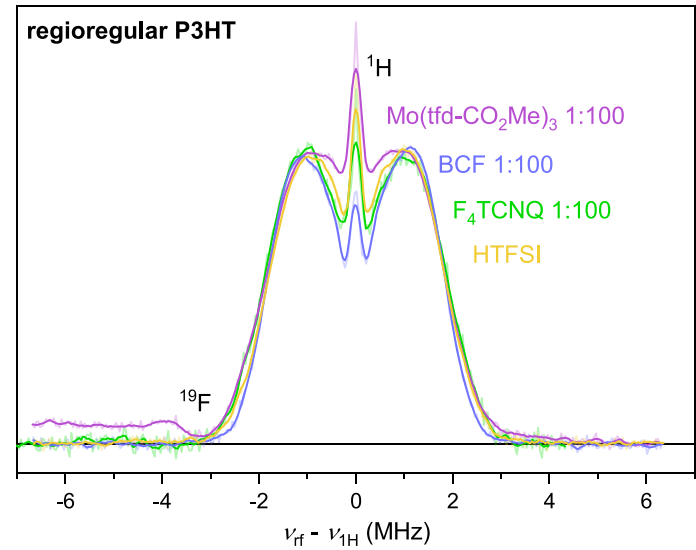

(b)

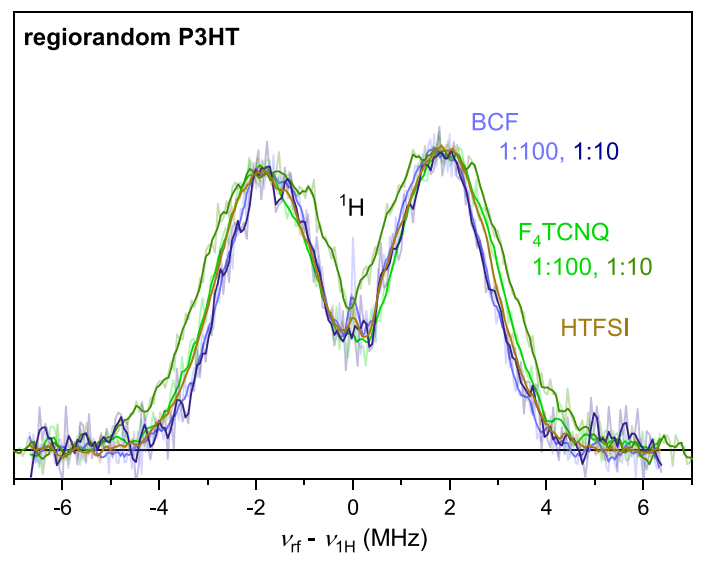

Figure 7. Q-band ${ }^{1} \mathrm{H}$ Davies ENDOR spectra recorded on frozen chloroform solutions of rreP3HT (a) and rraP3HT (b) doped with $\mathrm{F}_{4} \mathrm{TCNQ}, \mathrm{BCF}, \mathrm{HTFSI}$, or $\mathrm{Mo}\left(\mathrm{tfd}-\mathrm{CO}_{2} \mathrm{Me}\right)_{3}$ at a doping ratio of 1:100 (and 1:10 for $\mathrm{rraP} 3 \mathrm{HT}$ ) at a temperature of $10 \mathrm{~K}$. ENDOR spectra were recorded at the maximum of the EPR signal of the P3HT radical cation at a magnetic field around $1213.5 \mathrm{mT}$ (see SI section 6 for further experimental details). Lower signal-to-noise ratio for the spectra in (b) is due to the significantly smaller number of spincarrying moieties in doped rraP3HT solutions.

$2 a$ and 6a. Differences in the central region of the ENDOR spectra recorded for the different dopants are likely due to different distributions of distant, weakly coupled protons and, in the case of $\mathrm{F}_{4} \mathrm{TCNQ}$ and $\mathrm{Mo}\left(\mathrm{tfd}-\mathrm{CO}_{2} \mathrm{Me}\right)_{3}$, contributions from protons weakly coupled to the dopant anions arising from partial spectral overlap. In the case of $\mathrm{Mo}\left(\mathrm{tfd}-\mathrm{CO}_{2} \mathrm{Me}\right)_{3}$, additional contributions from fluorine couplings in the dopant radical anion can be observed around the ${ }^{19} \mathrm{~F}$ Larmor frequency (ca. $3 \mathrm{MHz}$ lower than the ${ }^{1} \mathrm{H}$ Larmor frequency at the magnetic field of the experiment). The ENDOR spectra corresponding to the small fraction of radical cations on rraP3HT generated by doping with $\mathrm{F}_{4} \mathrm{TCNQ} \mathrm{BCF}$, and HTFSI are significantly broader compared to those obtained for rreP3HT, indicating a decrease in the extent of delocalization. Following the approach described by Aguirre et al. for determination of the extent of spin delocalization from ${ }^{1} \mathrm{H}$ ENDOR spectra of $\mathrm{I}_{2}$-doped $\operatorname{rreP} 3 \mathrm{HT},{ }^{58}$ we estimated delocalization over about 12 thiophene rings in the case of rreP3HT, in agreement with their results, ${ }^{58}$ and a reduced delocalization over about 6 thiophene rings in the case of rraP3HT (see SI section 6 for details). Note that the signal 

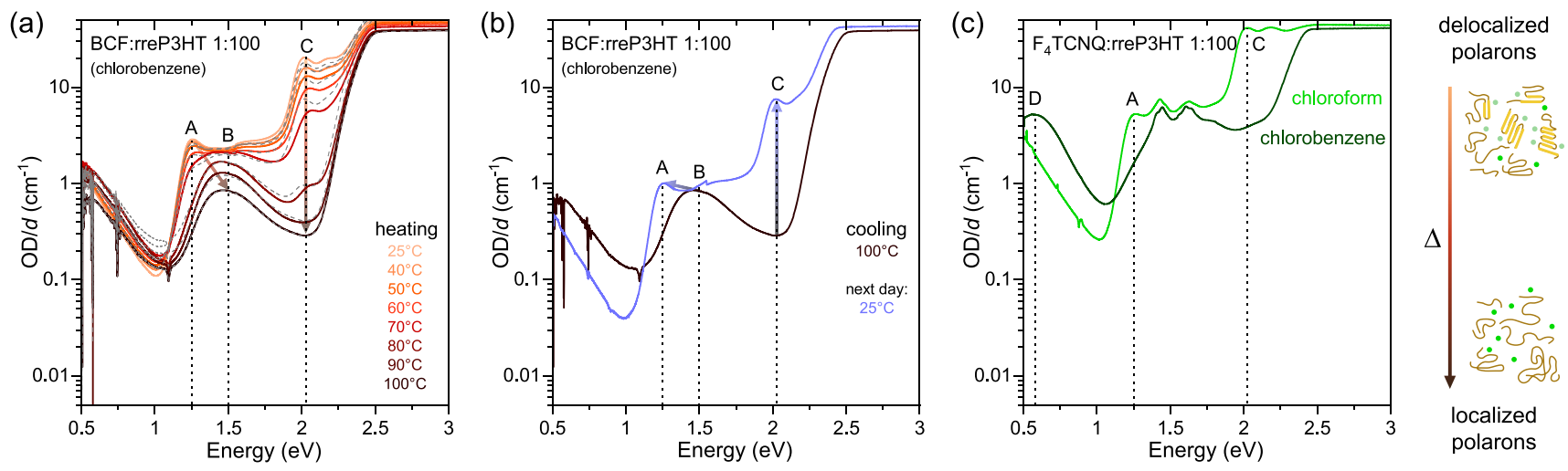

Figure 8. (a) Temperature-dependent UV-vis-NIR solution spectra of 1:100 BCF-doped rreP3HT in chlorobenzene, and (b) Spectra at 25 and $100{ }^{\circ} \mathrm{C}$. Dashed gray lines in (a) correspond to the spectra reconstructed as weighted sums of the spectra of polarons on aggregated and individual chains. (data in these two graphs have been shown and discussed in ref 19). (c) Comparison of the UV-vis-NIR absorption spectra of 1:100 $\mathrm{F}_{4}$ TCNQ-doped rreP3HT solution in chloroform (light green) vs chlorobenzene (dark green). Vertical dotted lines in the graphs highlight characteristic peak positions of the delocalized polaron (A), the localized polaron (B), the P3HT aggregate peak (C) and the $\mathrm{P}_{1}$ peak of the localized P3HT polaron in chlorobenzene (D).

strength of the doped rraP3HT samples is much weaker, implying that the concentration of these polarons is low and that polarons likely do not represent the main doping-induced species. These results support the hypothesis that doping with the ICT mechanism is favored in polymers permitting extensive charge delocalization, while in systems with limited charge delocalization such as in oligomers or strongly disordered polymers, inefficient charge-transfer mechanisms prevail.

\section{DISCUSSION}

To summarize the main results, we find that all three dopants $\left(\mathrm{F}_{4} \mathrm{TCNQ}, \mathrm{BCF}\right.$, and $\left.\mathrm{Mo}\left(\mathrm{tfd}-\mathrm{CO}_{2} \mathrm{Me}\right)_{3}\right)$ introduce essentially identical polaronic species in rreP3HT in chloroform solution, as indicated by the close agreement of the molar extinction coefficient spectra in Figure 6a, the EPR spectra in Figure 4a, and the ENDOR spectra in Figure 7a. The excellent agreement of the optical spectra in shape, oscillator strength, and peak position rules out a strong contribution from other dopinginduced species to the NIR optical absorption. It has been previously shown that in BCF-doped rreP3HT solutions, polarons on nonaggregated chains contribute moderately to the NIR absorption spectrum (depending on the doping ratio and the polymer concentration). ${ }^{19}$ On the other hand, Mo(tfd$\left.\mathrm{CO}_{2} \mathrm{Me}\right)_{3}$ does not induce such localized polarons under any of the conditions studied here (see Figure S4 and below). Therefore, the overall contribution of localized polarons to the extinction spectra in Figure 6a must be very small. Other possible species contributing to the NIR absorption may be bipolarons. As per spectroelectrochemistry results in the literature, bipolarons exhibit a broad spectrum with a continuous increase of the absorption strength with decreasing photon energy and a low-energy absorption peak at around 0.7 eV. ${ }^{71,72}$ Such a contribution is inconsistent with the distinct shape of the optical absorption spectra in Figure 6a. We, therefore, assign the NIR optical absorption of our doped rreP3HT samples in chloroform entirely to delocalized polarons on P3HT aggregates, irrespective of the choice of the dopant molecule.

On the other hand, our experimental results hint at the occurrence of different interactions between the dopants and
rreP3HT. For example, EPR shows the presence of an exchange interaction of the $\mathrm{P} 3 \mathrm{HT}$ radical cation with the $\mathrm{F}_{4} \mathrm{TCNQ}$ anion, whereas there is no evidence of such strong interactions of the spin-carrying species in the case of the other two dopants. The lack of a second spin-carrying species in the BCF-doped solutions differs from the situation proposed for PCPDTBT $^{36}$ but may reflect a similar doping mechanism with an additional step involving $\mathrm{H}_{2}$ loss. Yet, the NIR absorption spectra are identical for all three dopants in solution. This is in contrast to solid doped rreP3HT layers, which often reveal differences not only in the exact shape of the $\mathrm{P}_{2}$ double-peak feature but also in the position of the $\mathrm{P}_{1}$ transition, depending on the doping concentration and conditions. ${ }^{22}$ Given the absence of these effects in our solution studies, our data suggest that the exact nature and the optical absorption of the doping-induced polarons on rreP3HT in the solid state is influenced by mechanical strain and the unavoidable heterogeneity of the solid films. It has been shown recently that the broad double-peaked $\mathrm{P}_{2}$ transition is due to polarons on aggregated polymer chains. ${ }^{19}$ The presence of the solvent will allow these aggregates to relieve any mechanical stress and adopt a thermodynamically stable conformation, which then enables the formed polarons to delocalize along, and possibly among, the neighboring polymer backbones.

In rraP3HT, on the other hand, the type of species formed depends largely on the choice of the dopant. The planar $\mathrm{F}_{4} \mathrm{TCNQ}$ predominantly generates a CTC with the polymer, whereas doping with BCF or HTFSI yields a low concentration of P3HT polarons, which are more localized compared to that in aggregated rreP3HT. The preference for CTC complex formation in $\mathrm{F}_{4} \mathrm{TCNQ}: \mathrm{rraP} 3 \mathrm{HT}$ is consistent with the higher IE of rra- vs rreP3HT. The bulky $\mathrm{Mo}\left(\mathrm{tfd}-\mathrm{CO}_{2} \mathrm{Me}\right)_{3}$ is consistently found to not dope rraP3HT, in agreement with electron affinity and electrochemical data, while CTC formation is likely precluded by the bulky $3 \mathrm{D}$ shape of the dopant molecule. The absence of a peak at $2 \mathrm{eV}$ (assigned to doping-induced chain aggregation ${ }^{19}$ ) in any of the doped rraP3HT solutions shows that the rraP3HT chains are unable to planarize and form aggregates.

rreP3HT and rraP3HT represent extreme cases. This raises the question of whether other doping-induced species can form 
under other conditions. Additionally, given the fact that the rreP3HT chains in solution are also in the twisted (nonaggregated) conformation prior to addition of a dopant, one may ask whether a species similar to that observed in raP3HT solutions is a necessary precursor to the delocalized polarons on aggregated rreP3HT. On the same note, one might wonder whether the different species (delocalized polaron, localized polaron, and CTC) may be interconverted when deliberately changing the degree of chain aggregation. Finally, the low doping efficiency in raP3HT is often attributed to the inability of the rraP3HT chain to planarize/aggregate and thereby delocalize the polaron, ${ }^{17}$ which in turn suggests that polarons cannot be efficiently formed at a high density on individual, nonaggregated chains. To address these questions, we complemented our study by performing UV-vis-NIR absorption and EPR experiments on doped rreP3HT solutions as a function of temperature. Increasing the temperature increases the solubility of the compounds due to entropic effects while also providing thermal energy to induce a larger twist of the backbone and reduce the effective conjugation length.

We previously reported temperature-dependent absorption spectra of 1:100 BCF-doped rreP3HT in chlorobenzene (CB) solution, ${ }^{37} \mathrm{CB}$ being chosen because of its higher boiling point. The characteristic changes of the optical absorption features upon heating have been interpreted in terms of a temperatureinduced deaggregation of the P3HT chains. ${ }^{26,73}$ Figure $8 \mathrm{a}$ and $8 \mathrm{~b}$ shows temperature-dependent measurements on 1:100 BCF-doped rreP3HT solutions (selected spectra from this experiment are also shown and discussed in ref 19). In agreement with the earlier interpretation, comparison to the spectra in Figure $6 \mathrm{a}$ and $6 \mathrm{~b}$ shows that polarons on aggregated P3HT dominate the absorption at room temperature, while the absorption at $100{ }^{\circ} \mathrm{C}$ is entirely determined by the more localized polaron on isolated chains (Figure S16). Notably, the spectra at intermediate temperatures can be fitted as a superposition of these species showing the coexistence of polarons on aggregated and individual chains (Figure 8a and Figure S17) with no evidence for any other species being formed. Increasing the temperature leads to an increase in the contribution from the latter at the expense of the former (see Table S4). Cooling of the solution from $100{ }^{\circ} \mathrm{C}$ is accompanied by a slow recovery of the initial absorption peaks, although the process is not completely reversible. This is consistent with the notion of an irreversible reaction of the formed dopant species.

We observe a similar behavior while heating a 1:100 $\mathrm{F}_{4}$ TCNQ-doped rreP3HT solution in CF (see Figure S18), which also undergoes rapid dedoping upon heating. Cooling the solution back to room temperature restores the original spectrum, showing that doping and dedoping of $\mathrm{P} 3 \mathrm{HT}$ with $\mathrm{F}_{4}$ TCNQ is fully reversible, ${ }^{74}$ in contrast to the case of BCF doping as discussed above.

Interestingly, the absorption spectrum of $\mathrm{F}_{4} \mathrm{TCNQ}$-rreP3HT in $\mathrm{CB}$ is dominated by the characteristic absorption features of a polaron on nonaggregated chains even at room temperature, as confirmed by the near absence of the P3HT aggregate peak at $2 \mathrm{eV}$ and the spectral location of the $\mathrm{P}_{1}$ transition at $0.6 \mathrm{eV}$ (see Figure $8 \mathrm{c}$ ). Our spectra are reminiscent of the results of optical absorption measurements on $\mathrm{F}_{4}$ TCNQ-doped rreP3HT in CB by Wang et al., ${ }^{26}$ where the NIR absorption was, however, attributed to P3HT bipolarons. Absorption spectroscopy by Müller et al. suggested a higher degree of aggregation (and stronger doping) in CB solution (compared to CF). ${ }^{32}$ These experiments were, however, conducted at 50 ${ }^{\circ} \mathrm{C}$ and lower polymer concentrations $\left(0.012 \mathrm{mg} \mathrm{mL}^{-1}\right)$. Previous studies ${ }^{19,26,27,73}$ as well as the results presented in this work demonstrate that the solution temperature and polymer concentration affect the degree of aggregation and the type and concentration of doping-induced species. Hence, we believe that at room temperature and intermediate polymer concentrations of $2 \mathrm{mg} \mathrm{mL}^{-1}, \mathrm{~F}_{4}$ TCNQ-doped rreP3HT chains are more solvated in $\mathrm{CB}$ than in $\mathrm{CF}$. The NIR region of the roomtemperature optical spectrum can be reconstructed by considering contributions from the delocalized and the localized polaron absorption as ca. $3 \%$ and $23 \%$ of the original $\mathrm{F}_{4} \mathrm{TCNQ}$ dopant concentration, respectively. This agrees very well with the quantitative EPR results which show that ca. $20 \pm$ $1 \%$ of the employed dopants yield polarons on P3HT (Figure S19). Heating the $\mathrm{F}_{4}$ TCNQ-rreP3HT solution in CB causes a sudden decrease of the absorption strength at ca. $70{ }^{\circ} \mathrm{C}$ while not affecting the shape of the spectrum (Figure S20a). At the same time, the spin concentration measured by EPR exhibits a sharp drop (see Figure S20b and S20c). Cooling back to room temperature restores the initial spectrum, implying that the process of doping and dedoping with $\mathrm{F}_{4} \mathrm{TCNQ}$ is reversible. However, in contrast to the heating scan, the characteristic absorption of the CTC can be nicely discerned at $35-45{ }^{\circ} \mathrm{C}$ in the cooling cycle. Analysis of the NIR region reveals that the spectra in this temperature range consist almost entirely of CTC with up to $40 \%$ of the added dopants forming CTCs (Figure S21b). At lower temperatures $\left(<35{ }^{\circ} \mathrm{C}\right)$, this signal is superimposed on the absorption signal from localized polarons and is accompanied by a progressive reduction of the CTC contribution. Notably, the heating-cooling cycle of the 1:100 $\mathrm{F}_{4}$ TCNQ:rreP3HT system in $\mathrm{CB}$ exhibits a pronounced hysteresis. While the absorption from the localized polaron is stable upon heating up to a temperature of $60^{\circ} \mathrm{C}$, it is only at $30{ }^{\circ} \mathrm{C}$ in the cooling cycle that a strong polaron signal reappears (see also the result from EPR in Figure 20b). Given that the restoration of this species follows the appearance of the CTC, we speculate that the CTC is indeed the precursor to localized polarons in $\mathrm{F}_{4} \mathrm{TCNQ}$-doped rreP3HT.

The situation is once again different when heating and cooling a rreP3HT solution doped with $\mathrm{Mo}\left(\mathrm{tfd}-\mathrm{CO}_{2} \mathrm{Me}\right)_{3}$ in CF (Figure S22). Here, the NIR spectra show only the contribution from the delocalized polaron on the $\mathrm{P} 3 \mathrm{HT}$ aggregates with the absorption strength depending on temperature and time. In agreement with the slow polaron formation at room temperature (Figure S4a), the polaron signal is restored only slowly upon cooling, suggesting an energetic/kinetic barrier for polaron formation. Note that we did not find significant doping-induced NIR absorption signals in $\mathrm{Mo}\left(\mathrm{tfd}-\mathrm{CO}_{2} \mathrm{Me}\right)_{3}$-doped rreP3HT solutions in $\mathrm{CB}$.

\section{CONCLUSIONS}

In conclusion, we used optical, FTIR, and EPR spectroscopies to qualitatively and quantitatively evaluate the doping mechanism of rreP3HT and rraP3HT solutions with $\mathrm{F}_{4} \mathrm{TCNQ}, \mathrm{BCF}$, and $\mathrm{Mo}\left(\mathrm{tfd}-\mathrm{CO}_{2} \mathrm{Me}\right)_{3}$. This allowed us to identify three distinct optical signatures of $\mathrm{P} 3 \mathrm{HT}$, assigned to delocalized polarons (ICT), localized/isolated $\mathrm{P} 3 \mathrm{HT}$ polarons, and charge-transfer complexes, and estimate their molar extinction coefficient values. Our high-resolution UV-visNIR and EPR measurements confirmed that all three dopants consistently form the same predominant species (i.e., 
delocalized polarons) in rreP3HT solution, wherein the chains are free to aggregate. However, when the ability of the polymer chains to aggregate is suppressed, (e.g., due to steric hindrance as in raP3HT or at higher temperatures) the polymer-dopant interactions become more critical, as reflected by the reduced doping (ionization) efficiency, and/or CTC formation. This was further demonstrated by the proton hyperfine couplings measured by ENDOR spectroscopy, which showed that doped rreP3HT indeed exhibits a more extensive spin delocalization when compared to doped rraP3HT. Thus, the local morphology in the polymer chain is a more crucial parameter in determining the doping mechanism than the shape or size of the dopant molecule. On a broader scale, our findings demonstrate the close interplay between the dopant, the chain conformation, and the microstructure on the outcome of the semiconductor/dopant reaction.

\section{ASSOCIATED CONTENT}

\section{SI Supporting Information}

The Supporting Information is available free of charge at https://pubs.acs.org/doi/10.1021/acs.jpcb.0c03517.

Additional UV-vis-NIR spectra showing spectral changes as a function of time and light exposure, details on the quantitative analysis of the UV-vis-NIR spectra, FTIR data, additional EPR data, simulations and experimental details, temperature-dependent UV-vis and EPR data, details on the TDDFT calculations (PDF)

\section{AUTHOR INFORMATION}

\section{Corresponding Author}

Dieter Neher - Institut für Physik und Astronomie, Universität Potsdam, 14476 Potsdam, Germany; 이이.org/0000-00016618-8403; Email: neher@uni-potsdam.de

\section{Authors}

Malavika Arvind - Institut für Physik und Astronomie, Universität Potsdam, 14476 Potsdam, Germany

Claudia E. Tait - Institut für Experimentalphysik, Berlin Joint EPR Lab, Freie Universität Berlin, 14195 Berlin, Germany;

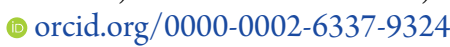

Michele Guerrini - Institut für Physik and IRIS Adlershof, Humboldt-Universität zu Berlin, 12489 Berlin, Germany; Institut für Physik, Carl von Ossietzky Universität Oldenburg, 26129 Oldenburg, Germany

Jannis Krumland - Institut für Physik and IRIS Adlershof, Humboldt-Universität zu Berlin, 12489 Berlin, Germany

Ana M. Valencia - Institut für Physik and IRIS Adlershof, Humboldt-Universität zu Berlin, 12489 Berlin, Germany; Institut für Physik, Carl von Ossietzky Universität Oldenburg, 26129 Oldenburg, Germany; (1) orcid.org/0000-0003-00953680

Caterina Cocchi - Institut für Physik and IRIS Adlershof, Humboldt-Universität zu Berlin, 12489 Berlin, Germany; Institut für Physik, Carl von Ossietzky Universität Oldenburg, 26129 Oldenburg, Germany

Ahmed E. Mansour - Institut für Physik and IRIS Adlershof, Humboldt-Universität zu Berlin, 12489 Berlin, Germany; Helmholtz-Zentrum Berlin für Materialien und Energie GmbH, 12489 Berlin, Germany

Norbert Koch - Institut für Physik and IRIS Adlershof, Humboldt-Universität zu Berlin, 12489 Berlin, Germany;
Helmholtz-Zentrum Berlin für Materialien und Energie GmbH, 12489 Berlin, Germany; 10 orcid.org/0000-0002-6042-6447

Stephen Barlow - School of Chemistry and Biochemistry and Center for Organic Photonics and Electronics, Georgia Institute of Technology, Atlanta, Georgia 30332-0400, United States; (1) orcid.org/0000-0001-9059-9974

Seth R. Marder - School of Chemistry and Biochemistry and Center for Organic Photonics and Electronics, Georgia Institute of Technology, Atlanta, Georgia 30332-0400, United States; (1) orcid.org/0000-0001-6921-2536

Jan Behrends - Institut für Experimentalphysik, Berlin Joint EPR Lab, Freie Universität Berlin, 14195 Berlin, Germany; (1) orcid.org/0000-0003-1024-428X

Complete contact information is available at:

https://pubs.acs.org/10.1021/acs.jpcb.0c03517

\section{Author Contributions}

${ }^{\S}$ M.A. and C.E.T.: These authors contributed equally to this work.

\section{Notes}

The authors declare no competing financial interest.

\section{ACKNOWLEDGMENTS}

This work was funded by the German Research Foundation (DFG) through the project "FoMEDOS" (Project number: 286798544) and the National Science Foundation through the DMREF Program (DMR-1729737). C.E.T. would like to acknowledge financial support through a Marie Curie Individual Fellowship (H2020-MSCA-IF-743419). Computational resources were provided by the North-German Supercomputing Alliance (HLRN), project bep00076. We also thank Bowen Sun (University of Potsdam) for complementary measurements, Kelvin Yao (Freie Universität Berlin) for his help in the early stages of the experiments, and Yadong Zhang for the synthesis of $\mathrm{Mo}\left(\mathrm{tfd}-\mathrm{CO}_{2} \mathrm{Me}\right)_{3}$.

\section{REFERENCES}

(1) Ostroverkhova, O. Handbook of Organic Materials for Optical and (Opto)Electronic Devices: Properties and Applications; Elsevier Science, 2013.

(2) Walzer, K.; Maennig, B.; Pfeiffer, M.; Leo, K. Highly Efficient Organic Devices Based on Electrically Doped Transport Layers. Chem. Rev. 2007, 107, 1233-1271.

(3) Pingel, P.; Neher, D. Comprehensive Picture of p-Type Doping of P3HT with the Molecular Acceptor F4TCNQ. Phys. Rev. B: Condens. Matter Mater. Phys. 2013, 87, 115209.

(4) Salzmann, I.; Heimel, G.; Duhm, S.; Oehzelt, M.; Pingel, P.; George, B. M.; Schnegg, A.; Lips, K.; Blum, R.-P.; Vollmer, A.; et al. Intermolecular Hybridization Governs Molecular Electrical Doping. Phys. Rev. Lett. 2012, 108, 035502.

(5) Lüssem, B.; Riede, M.; Leo, K. Doping of Organic Semiconductors. Phys. Status Solidi A 2013, 210, 9-43.

(6) Jacobs, I. E.; Moulé, A. J. Controlling Molecular Doping in Organic Semiconductors. Adv. Mater. 2017, 29, 1703063.

(7) Aziz, E. F.; Vollmer, A.; Eisebitt, S.; Eberhardt, W.; Pingel, P.; Neher, D.; Koch, N. Localized Charge Transfer in a Molecularly Doped Conducting Polymer. Adv. Mater. 2007, 19, 3257-3260.

(8) Gao, J.; Roehling, J. D.; Li, Y.; Guo, H.; Moulé, A. J.; Grey, J. K. The Effect of 2,3,5,6-Tetrafluoro-7,7,8,8-Tetracyanoquinodimethane Charge Transfer Dopants on the Conformation and Aggregation of Poly(3-hexylthiophene). J. Mater. Chem. C 2013, 1, 5638-5646.

(9) Duong, D. T.; Wang, C.; Antono, E.; Toney, M. F.; Salleo, A. The Chemical and Structural Origin of Efficient p-Type Doping in P3HT. Org. Electron. 2013, 14, 1330-1336. 
(10) Méndez, H.; Heimel, G.; Winkler, S.; Frisch, J.; Opitz, A.; Sauer, K.; Wegner, B.; Oehzelt, M.; Röthel, C.; Duhm, S.; et al. Charge-Transfer Crystallites as Molecular Electrical Dopants. Nat. Commun. 2015, 6, 8560.

(11) Hamidi-Sakr, A.; Biniek, L.; Bantignies, J. L.; Maurin, D.; Herrmann, L.; Leclerc, N.; Lévêque, P.; Vijayakumar, V.; Zimmermann, N.; Brinkmann, M. A Versatile Method to Fabricate Highly In-Plane Aligned Conducting Polymer Films with Anisotropic Charge Transport and Thermoelectric Properties: The Key Role of Alkyl Side Chain Layers on the Doping Mechanism. Adv. Funct. Mater. 2017, 27, 1700173.

(12) Österbacka, R.; An, C. P.; Jiang, X. M.; Vardeny, Z. V. TwoDimensional Electronic Excitations in Self-Assembled Conjugated Polymer Nanocrystals. Science 2000, 287, 839-842.

(13) Khlaifia, D.; Ewels, C. P.; Massuyeau, F.; Chemek, M.; Faulques, E.; Duvail, J.-L.; Alimi, K. Unraveling the Real Structures of Solution-Based and Surface-Bound Poly(3-hexylthiophene) (P3HT) Oligomers: a Combined Theoretical and Experimental Study. RSC Adv. 2016, 6, 56174-56182.

(14) Gallaher, J. K.; Chen, K.; Huff, G. S.; Prasad, S. K. K.; Gordon, K. C.; Hodgkiss, J. M. Evolution of Nonmirror Image Fluorescence Spectra in Conjugated Polymers and Oligomers. J. Phys. Chem. Lett. 2016, 7, 3307-3312.

(15) Scharsich, C.; Lohwasser, R. H.; Sommer, M.; Asawapirom, U.; Scherf, U.; Thelakkat, M.; Neher, D.; Köhler, A. Control of Aggregate Formation in Poly(3-hexylthiophene) by Solvent, Molecular Weight, and Synthetic Method. J. Polym. Sci., Part B: Polym. Phys. 2012, 50, $442-453$.

(16) Yamamoto, T.; Komarudin, D.; Arai, M.; Lee, B.-L.; Suganuma, H.; Asakawa, N.; Inoue, Y.; Kubota, K.; Sasaki, S.; Fukuda, T.; et al. Extensive Studies on $\pi$-Stacking of Poly(3-alkylthiophene-2,5-diyl)s and Poly(4-alkylthiazole-2,5-diyl)s by Optical Spectroscopy, NMR Analysis, Light Scattering Analysis, and X-Ray Crystallography. J. Am. Chem. Soc. 1998, 120, 2047-2058.

(17) Gao, J.; Niles, E. T.; Grey, J. K. Aggregates Promote Efficient Charge Transfer Doping of Poly(3-hexylthiophene). J. Phys. Chem. Lett. 2013, 4, 2953-2957.

(18) Heimel, G. The Optical Signature of Charges in Conjugated Polymers. ACS Cent. Sci. 2016, 2, 309-315.

(19) Mansour, A. E.; Lungwitz, D.; Schultz, T.; Arvind, M.; Valencia, A. M.; Cocchi, C.; Opitz, A.; Neher, D.; Koch, N. The Optical Signatures of Molecular-Doping Induced Polarons in Poly(3hexylthiophene-2,5-diyl): Individual Polymer Chains versus Aggregates. J. Mater. Chem. C 2020, 8, 2870-2879.

(20) Sirringhaus, H.; Brown, P. J.; Friend, R. H.; Nielsen, M. M.; Bechgaard, K.; Langeveld-Voss, B. M. W.; Spiering, A. J. H.; Janssen, R. A. J.; Meijer, E. W.; Herwig, P.; et al. Two-Dimensional Charge Transport in Self-Organized, High-Mobility Conjugated Polymers. Nature 1999, 401, 685-688.

(21) Janzen, D. E.; Burand, M. W.; Ewbank, P. C.; Pappenfus, T. M.; Higuchi, H.; da Silva Filho, D. A.; Young, V. G.; Brédas, J.-L.; Mann, K. R. Preparation and Characterization of $\pi$-Stacking Quinodimethane Oligothiophenes. Predicting Semiconductor Behavior and Bandwidths from Crystal Structures and Molecular Orbital Calculations. J. Am. Chem. Soc. 2004, 126, 15295-15308.

(22) Scholes, D. T.; Yee, P. Y.; Lindemuth, J. R.; Kang, H.; Onorato, J.; Ghosh, R.; Luscombe, C. K.; Spano, F. C.; Tolbert, S. H.; Schwartz, B. J. The Effects of Crystallinity on Charge Transport and the Structure of Sequentially Processed $\mathrm{F}_{4}$ TCNQ-Doped Conjugated Polymer Films. Adv. Funct. Mater. 2017, 27, 1702654.

(23) Ghosh, R.; Luscombe, C. K.; Hambsch, M.; Mannsfeld, S. C. B.; Salleo, A.; Spano, F. C. Anisotropic Polaron Delocalization in Conjugated Homopolymers and Donor-Acceptor Copolymers. Chem. Mater. 2019, 31, 7033-7045.

(24) Yee, P. Y.; Scholes, D. T.; Schwartz, B. J.; Tolbert, S. H. Dopant-Induced Ordering of Amorphous Regions in Regiorandom P3HT. J. Phys. Chem. Lett. 2019, 10, 4929-4934.

(25) Guo, J.; Ohkita, H.; Benten, H.; Ito, S. Charge Generation and Recombination Dynamics in Poly(3-hexylthiophene)/Fullerene Blend
Films with Different Regioregularities and Morphologies. J. Am. Chem. Soc. 2010, 132, 6154-6164.

(26) Wang, C.; Duong, D. T.; Vandewal, K.; Rivnay, J.; Salleo, A. Optical Measurement of Doping Efficiency in Poly(3-hexylthiophene) Solutions and Thin Films. Phys. Rev. B: Condens. Matter Mater. Phys. 2015, 91, 085205.

(27) Jacobs, I. E.; Cendra, C.; Harrelson, T. F.; Bedolla Valdez, Z. I.; Faller, R.; Salleo, A.; Moulé, A. J. Polymorphism Controls the Degree of Charge Transfer in a Molecularly Doped Semiconducting Polymer. Mater. Horiz. 2018, 5, 655-660.

(28) Neelamraju, B.; Watts, K. E.; Pemberton, J. E.; Ratcliff, E. L. Correlation of Coexistent Charge Transfer States in $\mathrm{F}_{4} \mathrm{TCNQ}$-Doped P3HT with Microstructure. J. Phys. Chem. Lett. 2018, 9, 6871-6877.

(29) Thomas, E. M.; Davidson, E. C.; Katsumata, R.; Segalman, R. A.; Chabinyc, M. L. Branched Side Chains Govern Counterion Position and Doping Mechanism in Conjugated Polythiophenes. ACS Macro Lett. 2018, 7, 1492-1497.

(30) Welch, G. C.; Bazan, G. C. Lewis Acid Adducts of Narrow Band Gap Conjugated Polymers. J. Am. Chem. Soc. 2011, 133, 46324644.

(31) Zalar, P.; Kuik, M.; Henson, Z. B.; Woellner, C.; Zhang, Y.; Sharenko, A.; Bazan, G. C.; Nguyen, T.-Q. Increased Mobility Induced by Addition of a Lewis Acid to a Lewis Basic Conjugated Polymer. Adv. Mater. 2014, 26, 724-727.

(32) Müller, L.; Nanova, D.; Glaser, T.; Beck, S.; Pucci, A.; Kast, A. K.; Schröder, R. R.; Mankel, E.; Pingel, P.; Neher, D.; et al. ChargeTransfer-Solvent Interaction Predefines Doping Efficiency in pDoped P3HT Films. Chem. Mater. 2016, 28, 4432-4439.

(33) Dai, A.; Zhou, Y.; Shu, A. L.; Mohapatra, S. K.; Wang, H.; Fuentes-Hernandez, C.; Zhang, Y.; Barlow, S.; Loo, Y.-L.; Marder, S. $\mathrm{R}$; et al. Enhanced Charge-Carrier Injection and Collection Via Lamination of Doped Polymer Layers p-Doped with a SolutionProcessible Molybdenum Complex. Adv. Funct. Mater. 2014, 24, 2197-2204.

(34) Mohapatra, S. K.; Zhang, Y.; Sandhu, B.; Fonari, M. S.; Timofeeva, T. V.; Marder, S. R.; Barlow, S. Synthesis, Characterization, and Crystal Structures of Molybdenum Complexes of Unsymmetrical Electron-Poor Dithiolene Ligands. Polyhedron 2016, $116,88-95$.

(35) Gao, W.; Kahn, A. Controlled p-Doping of Zinc Phthalocyanine by Coevaporation with Tetrafluorotetracyanoquinodimethane: $\mathrm{A}$ Direct and Inverse Photoemission Study. Appl. Phys. Lett. 2001, 79, 4040-4042.

(36) Lawrence, E. J.; Oganesyan, V. S.; Wildgoose, G. G.; Ashley, A. E. Exploring the Fate of the Tris(Pentafluorophenyl)Borane Radical Anion in Weakly Coordinating Solvents. Dalton Trans. 2013, 42, $782-789$.

(37) Pingel, P.; Arvind, M.; Kölln, L.; Steyrleuthner, R.; Kraffert, F.; Behrends, J.; Janietz, S.; Neher, D. p-Type Doping of Poly(3hexylthiophene) with the Strong Lewis Acid Tris(pentafluorophenyl)borane. Adv. Electron. Mater. 2016, 2, 1600204.

(38) Yurash, B.; Cao, D. X.; Brus, V. V.; Leifert, D.; Wang, M.; Dixon, A.; Seifrid, M.; Mansour, A. E.; Lungwitz, D.; Liu, T. Towards Understanding the Doping Mechanism of Organic Semiconductors by Lewis Acids. Nat. Mater. 2019, 18, 1327.

(39) Doerrer, L. H.; Green, M. L. H. Oxidation of $\left[\mathrm{M}\left(\eta-\mathrm{C}_{5} \mathrm{H}_{5}\right)_{2}\right], \mathrm{M}$ $=\mathrm{Cr}, \mathrm{Fe}$ or $\mathrm{Co}$, by the New Brønsted Acid $\mathrm{H}_{2} \mathrm{O} \cdot \mathrm{B}\left(\mathrm{C}_{6} \mathrm{~F}_{5}\right)_{3}$ Yielding the Salts $\left[\mathrm{M}\left(\eta-\mathrm{C}_{5} \mathrm{H}_{5}\right)_{2}\right]^{+} \mathrm{A}^{-}$, where $\mathrm{A}^{-}=\left[\left(\mathrm{C}_{6} \mathrm{~F}_{5}\right)_{3} \mathrm{~B}(\mu\right.$-OH $) \mathrm{B}$ $\left.\left(\mathrm{C}_{6} \mathrm{~F}_{5}\right)_{3}\right]^{-}$or $\left[\left(\mathrm{C}_{6} \mathrm{~F}_{5}\right)_{3} \mathrm{BOH} \cdot \cdots \mathrm{H}_{2} \mathrm{OB}\left(\mathrm{C}_{6} \mathrm{~F}_{5}\right)_{3}\right]^{-}$. J. Chem. Soc., Dalton Trans. 1999, 4325-4329.

(40) Guan, Z.-L.; Kim, J. B.; Wang, H.; Jaye, C.; Fischer, D. A.; Loo, Y.-L.; Kahn, A. Direct Determination of the Electronic Structure of the Poly(3-hexylthiophene):phenyl-[6,6]- $\mathrm{C}_{61}$ Butyric Acid Methyl Ester Blend. Org. Electron. 2010, 11, 1779-1785.

(41) Ko, S.; Hoke, E. T.; Pandey, L.; Hong, S.; Mondal, R.; Risko, C.; Yi, Y.; Noriega, R.; McGehee, M. D.; Brédas, J.-L.; et al. Controlled Conjugated Backbone Twisting for an Increased OpenCircuit Voltage while Having a High Short-Circuit Current in 
Poly(hexylthiophene) Derivatives. J. Am. Chem. Soc. 2012, 134, $5222-5232$.

(42) Panidi, J.; Paterson, A. F.; Khim, D.; Fei, Z.; Han, Y.; Tsetseris, L.; Vourlias, G.; Patsalas, P. A.; Heeney, M.; Anthopoulos, T. D. Remarkable Enhancement of the Hole Mobility in Several Organic Small-Molecules, Polymers, and Small-Molecule:Polymer Blend Transistors by Simple Admixing of the Lewis Acid p-Dopant $\mathrm{B}\left(\mathrm{C}_{6} \mathrm{~F}_{5}\right)_{3}$. Adv. Sci. 2018, 5, 1700290.

(43) Eaton, G. R.; Eaton, S. S.; Barr, D. P.; Weber, R. T. Quantitative EPR; Springer-Verlag: Vienna, 2010.

(44) Frisch, M. J.; Trucks, G. W.; Schlegel, H. B.; Scuseria, G. E.; Robb, M. A.; Cheeseman, J. R.; Scalmani, G.; Barone, V.; Petersson, G. A.; Nakatsuji, H., et al. Gaussian 16; Gaussian: Wallingford, CT, 2016.

(45) Yanai, T.; Tew, D. P.; Handy, N. C. A New Hybrid ExchangeCorrelation Functional Using the Coulomb-Attenuating Method (CAM-B3LYP). Chem. Phys. Lett. 2004, 393, 51-57.

(46) Grimme, S.; Antony, J.; Ehrlich, S.; Krieg, H. A Consistent and Accurate $\mathrm{Ab}$ Initio Parametrization of Density Functional Dispersion Correction (DFT-D) for the 94 Elements H-Pu. J. Chem. Phys. 2010, 132, 154104.

(47) Cammi, R.; Mennucci, B. Linear Response Theory for the Polarizable Continuum Model. J. Chem. Phys. 1999, 110, 9877.

(48) Dixon, D. A.; Calabrese, J. C.; Miller, J. S. Crystal and Molecular Structure of the 2:1 Charge-Transfer Salt of Decamethylferrocene and Perfluoro-7,7,8,8-Tetracyano-p-Quinodimethane: [[Fe$\left.\left.\left(\mathrm{C}_{5} \mathrm{Me}_{5}\right)_{2}\right]^{++}\right]_{2}\left[\mathrm{TCNQF}_{4}\right]^{2-}$. The Electronic Structure of $\left[\mathrm{TCNQF}_{4}\right]_{\mathrm{n}}$ $(\mathrm{n}=0,1-, 2-)$. J. Phys. Chem. 1989, 93, 2284-2291.

(49) Kwaan, R. J.; Harlan, C. J.; Norton, J. R. Generation and Characterization of the Tris(pentafluorophenyl)borane Radical Anion. Organometallics 2001, 20, 3818-3820.

(50) Jacobs, I. E.; Aasen, E. W.; Oliveira, J. L.; Fonseca, T. N.; Roehling, J. D.; Li, J.; Zhang, G.; Augustine, M. P.; Mascal, M.; Moule, A. J. Comparison of Solution-Mixed and Sequentially Processed P3HT:F4TCNQ Films: Effect of Doping-Induced Aggregation on Film Morphology. J. Mater. Chem. C 2016, 4, 3454-3466.

(51) Ye, T.; Chen, W.; Jin, S.; Hao, S.; Zhang, X.; Liu, H.; He, D. Enhanced Efficiency of Planar Heterojunction Perovskite Solar Cells by a Light Soaking Treatment on Tris(pentafluorophenyl)boraneDoped Poly(triarylamine) Solution. ACS Appl. Mater. Interfaces 2019, 11, 14004-14010.

(52) Valencia, A. M.; Cocchi, C. Electronic and Optical Properties of Oligothiophene-F4TCNQ Charge-Transfer Complexes: The Role of the Donor Conjugation Length. J. Phys. Chem. C 2019, 123, 96179623.

(53) Valencia, A. M.; Guerrini, M.; Cocchi, C. Ab Initio Modelling of Local Interfaces in Doped Organic Semiconductors. Phys. Chem. Chem. Phys. 2020, 22, 3527-3538.

(54) Fuzell, J.; Jacobs, I. E.; Ackling, S.; Harrelson, T. F.; Huang, D. M.; Larsen, D.; Moulé, A. J. Optical Dedoping Mechanism for P3HT:F4TCNQ Mixtures. J. Phys. Chem. Lett. 2016, 7, 4297-4303. (55) Cocchi, C.; Draxl, C. Bound Excitons and Many-Body Effects in X-Ray Absorption Spectra of Azobenzene-Functionalized SelfAssembled Monolayers. Phys. Rev. B: Condens. Matter Mater. Phys. 2015, 92, 205105.

(56) Cocchi, C.; Draxl, C. Optical Spectra from Molecules to Crystals: Insight from Many-Body Perturbation Theory. Phys. Rev. B: Condens. Matter Mater. Phys. 2015, 92, 205126.

(57) Guerrini, M.; Cocchi, C.; Calzolari, A.; Varsano, D.; Corni, S. Interplay between Intra- and Intermolecular Charge Transfer in the Optical Excitations of J-Aggregates. J. Phys. Chem. C 2019, 123, 6831-6838.

(58) Aguirre, A.; Gast, P.; Orlinskii, S.; Akimoto, I.; Groenen, E. J. J.; El Mkami, H.; Goovaerts, E.; Van Doorslaer, S. Multifrequency EPR Analysis of the Positive Polaron in $\mathrm{I}_{2}$-Doped Poly(3-hexylthiophene) and in Poly[2-methoxy-5-(3,7-dimethyloctyloxy)]-1,4-Phenylenevinylene. Phys. Chem. Chem. Phys. 2008, 10, 7129-7138.
(59) Sproules, S.; Banerjee, P.; Weyhermüller, T.; Yan, Y.; Donahue, J. P.; Wieghardt, K. Monoanionic Molybdenum and Tungsten Tris(dithiolene) Complexes: A Multifrequency EPR Study. Inorg. Chem. 2011, 50, 7106-7122.

(60) Fekl, U.; Sarkar, B.; Kaim, W.; Zimmer-De Iuliis, M.; Nguyen, N. Tuning of the Spin Distribution between Ligand- and Metal-Based Spin: Electron Paramagnetic Resonance of Mixed-Ligand Molybdenum Tris(dithiolene) Complex Anions. Inorg. Chem. 2011, 50, 86858687.

(61) Tomkiewicz, Y.; Taranko, A. R.; Torrance, J. B. Spin Susceptibility of Tetrathiafulvalene Tetracyanoquinodimethane, TTF-TCNQ in the Semiconducting Regime: Comparison with Conductivity. Phys. Rev. B 1977, 15, 1017-1023.

(62) Nordio, P.; Soos, Z.; McConnell, H. M. Spin Excitations in Ionic Molecular Crystals. Annu. Rev. Phys. Chem. 1966, 17, 237-260.

(63) Hoffman, B. M.; Hughes, R. ESR of TMPD-TCNQ: Spin Excitations of the Heisenberg Regular Linear Chain. J. Chem. Phys. 1970, 52, 4011-4023.

(64) Hofmann, A. I.; Kroon, R.; Yu, L.; Müller, C. Highly Stable Doping of a Polar Polythiophene through Co-Processing with Sulfonic Acids and Bistriflimide. J. Mater. Chem. C 2018, 6, 69056910.

(65) Valencia, D.; Whiting, G. T.; Bulo, R. E.; Weckhuysen, B. M. Protonated Thiophene-based Oligomers as Formed within Zeolites: Understanding Their Electron Delocalization and Aromaticity. Phys. Chem. Chem. Phys. 2016, 18, 2080-2086.

(66) Meneghetti, M.; Pecile, C. Charge-Transfer Organic Crystals: Molecular Vibrations and Spectroscopic Effects of ElectronMolecular Vibration Coupling of the Strong Electron Acceptor TCNQF4. J. Chem. Phys. 1986, 84, 4149-4162.

(67) Pingel, P.; Zhu, L.; Park, K. S.; Vogel, J.-O.; Janietz, S.; Kim, E.G.; Rabe, J. P.; Brédas, J.-L.; Koch, N. Charge-Transfer Localization in Molecularly Doped Thiophene-Based Donor Polymers. J. Phys. Chem. Lett. 2010, 1, 2037-2041.

(68) Ghani, F.; Opitz, A.; Pingel, P.; Heimel, G.; Salzmann, I.; Frisch, J.; Neher, D.; Tsami, A.; Scherf, U.; Koch, N. Charge Transfer in and Conductivity of Molecularly Doped Thiophene-Based Copolymers. J. Polym. Sci., Part B: Polym. Phys. 2015, 53, 58-63.

(69) Niklas, J.; Mardis, K. L.; Banks, B. P.; Grooms, G. M.; Sperlich, A.; Dyakonov, V.; Beaupre, S.; Leclerc, M.; Xu, T.; Yu, L.; et al. Highly-Efficient Charge Separation and Polaron Delocalization in Polymer-Fullerene Bulk-Heterojunctions: a Comparative MultiFrequency EPR and DFT Study. Phys. Chem. Chem. Phys. 2013, 15, 9562-9574.

(70) Steyrleuthner, R.; Zhang, Y.; Zhang, L.; Kraffert, F.; Cherniawski, B. P.; Bittl, R.; Briseno, A. L.; Brédas, J.-L.; Behrends, J. Impact of Morphology on Polaron Delocalization in a Semicrystalline Conjugated Polymer. Phys. Chem. Chem. Phys. 2017, 19, 36273639.

(71) Ratcliff, E. L.; Lee, P. A.; Armstrong, N. R. Work Function Control of Hole-Selective Polymer/ITO Anode Contacts: an Electrochemical Doping Study. J. Mater. Chem. 2010, 20, 2672-2679.

(72) Enengl, C.; Enengl, S.; Pluczyk, S.; Havlicek, M.; Lapkowski, M.; Neugebauer, H.; Ehrenfreund, E. Doping-Induced Absorption Bands in P3HT: Polarons and Bipolarons. ChemPhysChem 2016, 17, 3836-3844.

(73) Duong, D. T.; Phan, H.; Hanifi, D.; Jo, P. S.; Nguyen, T.-Q.; Salleo, A. Direct Observation of Doping Sites in TemperatureControlled, p-Doped P3HT Thin Films by Conducting Atomic Force Microscopy. Adv. Mater. 2014, 26, 6069-6073.

(74) Jacobs, I. E.; Wang, F.; Hafezi, N.; Medina-Plaza, C.; Harrelson, T. F.; Li, J.; Augustine, M. P.; Mascal, M.; Moulé, A. J. Quantitative Dedoping of Conductive Polymers. Chem. Mater. 2017, 29, 832-841. 